Accepted to ApJ: October 1, 2009

Preprint typeset using $\mathrm{IAT}_{\mathrm{E}} \mathrm{X}$ style emulateapj v. 08/22/09

\title{
FAR-ULTRAVIOLET DUST ALBEDO MEASUREMENTS IN THE UPPER SCORPIUS CLOUD USING THE SPINR SOUNDING ROCKET EXPERIMENT
}

\author{
N. K. Lewis, T. A. Cook, K. P. Wilton, S. Chakrabarti \\ Center for Space Physics and Department of Astronomy, Boston University, \\ Boston, MA 02215 \\ K. France \\ Canadian Institute for Theoretical Astrophysics, University of Toronto, \\ Toronto ON M5S 3H8 \\ AND \\ K. D. GORDON \\ Steward Observatory, University of Arizona, Tucson, AZ 85721 \\ ACCEPTED to ApJ: October 1, 2009
}

\begin{abstract}
The Spectrograph for Photometric Imaging with Numeric Reconstruction (SPINR) sounding rocket experiment was launched on 2000 August 4 to record far-ultraviolet (912-1450 A) spectral and spatial information for the giant reflection nebula in the Upper Scorpius region. The data were divided into three arbitrary bandpasses (912-1029 $\AA, 1030-1200 \AA$, and 1235-1450 $\AA$ ) for which stellar and nebular flux levels were derived. These flux measurements were used to constrain a radiative transfer model and to determine the dust albedo for the Upper Scorpius region. The resulting albedos were $0.28 \pm 0.07$ for the $912-1029 \AA$ bandpass, $0.33 \pm 0.07$ for the $1030-1200 \AA$ bandpass, and $0.77 \pm 0.13$ for the 1235-1450 A bandpass.

Subject headings: dust, extinction - ISM: individual (Scorpius OB2) — radiative transfer — ultraviolet: ISM
\end{abstract}

\section{INTRODUCTION}

Dust in the interstellar medium (ISM) is central to many physical processes, such as star and planet formation. In the ultraviolet (UV), the properties of interstellar dust are largely gleaned from the interactions between dust and the electromagnetic radiation from UV bright stars. Typically, these interactions manifest themselves through the absorption and scattering of UV light. The reduction of starlight by dust is commonly referred to as extinction, which has been characterized in the Milky Way Galaxy by an idealized set of wavelength dependent 'extinction curves' (see the review by Fitzpatrick (2004)).

The light scattering properties of dust are characterized by two quantities: the albedo $(a)$ and the asymmetry parameter $(g)$. Both $a$ and $g$ are functions of the wavelength of the incident light. Previous studies have probed reflection nebulae, dark clouds, and diffuse galactic light in an attempt to characterize the scattering properties of interstellar dust from infrared (IR) to UV wavelengths (see the review by Gordon (2004)). Although these studies have contributed to the characterization of dust in the ISM, there is still much debate about dust radiative transfer models and the appropriateness of techniques used in measuring $a$ and $g$ for a given sight line (Mathis et al. 2002; Draine 2003; Zubko et al. 2004). Further observations of dusty environments throughout our galaxy and beyond, along with the continued refinement of current dust models are needed in order to en-

Electronic address: nkhoward@bump.bu.edu

Electronic address: france@cita.utoronto.ca

Electronic address: kgordon@as.arizona.edu hance our understanding.

The Upper Scorpius cloud is ideally suited for probing the scattering properties of dust grains. It is an internally illuminated reflection nebula, which makes the total nebular flux strongly dependent on $a$ and only weakly dependent on $g$ (Gordon et al. 1994). This nebular geometry will result in an accurate measurement of $a$, but it is likely that any measure of $g$ will have large uncertainties. The Upper Scorpius region was also found by Gaustad \& van Buren (1993) to have very little foreground dust, which would add to the uncertainty in the scattering properties of the nebula. By considering this region as a whole, a large sample of UV bright stars are available as illumination sources for the dust model. Multiple illuminating sources will reduce the model's dependence on the accuracy of any one star's derived properties. It was for these reasons that the nebula in Upper Scorpius was selected as the target for the Spectrograph for Photometric Imaging with Numeric Reconstruction (SPINR) sounding rocket mission (Cook et al. 2003, hereafter Paper I).

The SPINR experiment provides a unique look at the ISM in the FUV wavelength regime from 912-1450 A. By recording wide-field $\left(\sim 16^{\circ}\right)$ spectral and spatial information from a given target simultaneously, a complete picture of the dust properties in a region can be derived. Paper I presents the details of the SPINR experiment while its data have been used by Lewis et al. (2005, hereafter Paper II) to derive the extinction properties along several lines of sight in the Upper Scorpius Cloud. Paper II found that the Upper Scorpius region possesses a wide range of extinction properties, which makes it 
an excellent probe of the various dust environments seen throughout the Milky Way. Thus far, only a handful of studies have probed dust scattering properties shortward of Ly $\alpha(1216 \AA)$ (Witt et al. 1993; Burgh et al. 2002; Suiatha et al. 2005; Shalima et al. 2006; Sujatha et al. 2007). The SPINR data are unique in that spectra for the entire $16^{\circ}$ field-of-view (FOV) were recorded simultaneously without the FOV limitations of a traditional long slit or the spectral resolution limitations of traditional wide band filters. Currently, there are significant discrepancies between model predictions of dust albedo in the FUV and observations (Gordon 2004). The insights gained from the SPINR study of the Upper Scorpius cloud will help to paint a more complete picture of dust scattering properties in the FUV.

The goal of this paper is to present the determination of $a$ and $g$ values for dust in the Upper Scorpius region at the effective wavelengths $\lambda_{\text {eff }}=973 \AA, 1106 \AA$, and $1261 \AA$. Additionally, the Upper Scorpius data presented in Gordon et al. (1994) were revisited with an improved radiative transfer model to determine the value of $a$ in the STS-39 FUV camera (FUVCam) bandpasses, $\lambda_{e f f}=$ $1362 \AA$ and $1769 \AA$.

\section{OBSERVATIONS AND IMAGE RECONSTRUCTION}

On 2000 August 4, the SPINR rocket experiment was launched from the White Sands Missile Range to record both spectral and spatial information from the Upper Scorpius cloud in the FUV. Approximately $400 \mathrm{~s}$ of data were recorded at a $2^{\prime} .5$ RMS spatial resolution and $10 \AA$ spectral resolution. SPINR optically compresses the FOV into a single spatial dimension at each wavelength which in combination with the rotation of the rocket about its optical axis creates a three-dimensional (position, wavelength, and time) data cube. Extracting the stellar spectra from the SPINR data cube is a fairly simple operation. One must simply identify the celestial coordinates corresponding to the axis of rotation of SPINR, then predict the phase and amplitude of the sine curve traced out by each star over the duration of the observation (Paper I). The point spread function (PSF) of the instrument varies with wavelength and position, but is on average Gaussian in shape with a full-width halfmaximum of 10 pixels $\left(\sim 7^{\prime} .8\right)$. Data lying within the PSF of the predicted sine curve can then be collected and assigned to the proper source.

A similar process can be performed to reconstruct a spatial image of the SPINR data. Paper I reviews several types of algorithms that could be used in the image reconstruction process. In this study, the Pixon (Piña \& Puetter 1993) method of image reconstruction was used to create images of the Upper Scorpius region in three arbitrary wavelength bands: 912-1029 A, 1030-1200 $\AA$, and 1235-1450 А. Figure 1] shows the reconstructed image for each SPINR wavelength bands and an image from the STS-39 FUVCam experiment (G. R. Carruthers 2006 , private communication) for the $1230-1600 \AA$ wavelength range. The FOV in each of these images has been constrained to the inner $10^{\circ}$ of the the SPINR FOV for comparison purposes. The SPINR images are at a significantly lower spatial resolution than the raw SPINR data because of computational restrictions on the image reconstruction process. For this reason, the data anal- ysis presented here was performed on the raw SPINR sinogram data.

\section{DATA ANALYSIS}

Following the work of Gordon et al. (1994), there were several items that needed to be calculated in order to properly constrain the dust model. One of the key items is the nebular to stellar flux ratio $\left(F_{N} / F_{*}\right)$ for each wavelength band. This ratio will constrain the range of acceptable solutions for the albedo, $a$. The derivation of the total stellar flux, $F_{*}$, for each bandpass is detailed in section 3.2 . The basic properties of the stellar sources considered in this study are detailed in Table 11. The derivation of the total nebular flux, $F_{N}$, is outlined in section 3.3 . Table 2 presents the values of $F_{N} / F_{*}$ for each bandpass derived in this study. The model (further described in section 3.1) also requires estimates of the dereddened luminosity and the depth into the cloud of each stellar source, which are calculated by determining the extinction (section 3.2.1), $\mathrm{H}_{2}$ absorption (section 3.2.2), and $R_{V}$ parameter (section 3.2.3) along each line of sight.

\subsection{Dust Model}

The DIRTY Monte Carlo radiative transfer model (Gordon et al. 2001; Misselt et al. 2001) was used in this study to determine the $a$ and $g$ values that best reproduced the observed nebular surface brightness of the Upper Scorpius cloud. The geometric model of the Upper Scorpius cloud used is identical to that of Gordon et al. (1994) with a uniform sphere of dust with each star embedded to a depth equal to its measured optical depth at each wavelength modeled. This simplistic geometry is used as it is more important for the radiative transfer solution to have each star embedded to the correct optical depth, than to the correct physical depth. The effects of the known clumpy structure of the ISM on albedo determinations Mathis et al. (2002) are mitigated by the multiple illumination sources in the Upper Scorpius cloud. The multiple illumination sources mean that the computed scattered flux is relatively insensitive to the details of a single star's parameters (luminosity, optical depth, etc.), which could be affected by clumpy structures along its line of sight. This prescription for the radiative transfer model for Upper Scorpius was shown to be valid, but not necessarily unique, by the close correspondence between the models and observed images in Gordon et al. (1994).

\subsection{Stellar Flux}

The total stellar flux in each wavelength band was determined by identifying those stars in the SPINR FOV that are bright in the ultraviolet and simply summing their measured fluxes. These UV bright stars were selected from the Astronomical Netherlands Satellite $(A N S)$ catalog (Wesselius et al. 1982) based on two criteria: 1) they reside inside the $16^{\circ} \mathrm{SPINR}$ FOV. 2) at $1500 \AA$ the wide band $(\delta \lambda=149 \AA)$ magnitude is less than 6.0 mag. For the fourteen brightest stars, measurements of their reddened luminosities for each bandpass were easily extracted from the SPINR data by knowledge of their relative position. Swaths of 30 pixels around the predicted stellar sine curves were extracted from the raw 
SPINR data in order to collect all the stellar light within $\sim 6 \sigma$ of the central Gaussian stellar peak. Pixels where two or more stellar sine curves intersected were ignored. Those pixels not associated with a stellar line of sight were used to create a background spectrum that was removed from each of the stellar spectra. This background spectrum includes the average nebular flux in the region in addition to the instrument background and telluric emissions. The flux from the dimmest nineteen stars in SPINR's FOV (15-33 in Table 1) could not be disentangled from the nebular flux because of low spatial resolution and were estimated using available data and stellar models as described below. This flux amounts to less than $5 \%$ of the total stellar flux in each bandpass.

For the 912-1029 $\AA$ and 1030-1200 $\AA$ bandpasses, estimates of the reddened luminosities for the dimmest nineteen stars were made using the Kurucz model (Kurucz 1992) corresponding to the surface gravity (Straižys \& Kuriliene 1981) and effective temperature (de Jager \& Nieuwenhuijzen 1987) predicted from the MK spectral type and luminosity class of each star (Table 1). The Kurucz models were then converted into SPINR units (counts $\mathrm{s}^{-1}$ ) using the dereddened spectrum of $\pi$ Sco as a standard. However, in order to make an estimate of the expected reddened luminosity of each star, the amount of reddening along each line of sight had to be calculated. Details on the derivation of extinction curves, $\mathrm{H}_{2}$ attenuations, and ratios of total to selective extinction, $R_{V}$, can be found in the following sections. Stellar fluxes derived from the Kurucz models accounted for less than $5 \%$ of the total reddened stellar luminosity and less than $10 \%$ of the total dereddened stellar luminosity.

In the 1235-1450 $\AA$ bandpass, International Ultraviolet Explorer (IUE) data (Table 1) were converted into SPINR units (counts $\mathrm{s}^{-1}$ ) using the spectrum from $\pi$ Sco as a standard then summed over the bandpass to obtain an estimate of the reddened luminosity for the dimmest nineteen stars in the FOV. IUE data were also used to estimate the reddened luminosities of $\tau$ Sco and $\rho$ Sco in the 1235-1450 $\AA$ bandpass because their proximity to the edge of the detector prevented an accurate measurement in this lower sensitivity bandpass. Table 3 presents the reddened luminosities for each star that were used in calculating the total stellar flux $\left(F_{*}\right)$ in each bandpass.

\subsubsection{Extinction Curves}

The extinction curves of the form $k(\lambda-V)=E(\lambda-$ $V) / E(B-V)$ for $\pi$ Sco, $\beta$ Sco, $\delta$ Sco, $\sigma$ Sco, $\omega$ Sco, $\nu$ Sco, and $\rho$ Oph, which account for $\sim 65 \%$ of the total reddened stellar luminosity, were previously derived from SPINR data in Paper II. For the other stellar lines of sight in this study with lower signal-to-noise ratios, it proved difficult to derive a reliable measure of the extinction from the SPINR data in the $912 \AA$ to $1450 \AA$ wavelength range. However, as was shown in Paper II and Sofia et al. (2005), a fairly reliable prediction of extinction in the FUV along a given line of sight can be made by fitting a curve according to the parameterization of Fitzpatrick \& Massa (1988) to IUE data then extrapolating the curve out shortward of Ly $\alpha$. For each line of sight possessing an $E(B-V) \geq 0.1$, an extinction curve was derived from the IUE data listed in Ta- ble 1 using the pair comparison method outlined in Paper II. The comparison stars used and the subsequent Fitzpatrick \& Massa (1988) parameterization for the extinction curves are found in Table 4. For lines of sight with $E(B-V)<0.1$ it was assumed that the Galactic mean curve could be used to deredden the data with the introduction of only small errors [less than $1 \%$ of $k(\lambda-V)$; (Green et al. 1992)].

\subsection{2. $\mathrm{H}_{2}$ Absorption}

When working with spectral data in the wavelength regime between the Lyman edge (912 $\AA$ ) and Ly $\alpha$ (1216 $\AA)$ it is important to consider the effects of $\mathrm{H}_{2}$ absorption. In addition to the attenuation of starlight by dust in this wavelength regime, the $\mathrm{H}_{2}$ Lyman and Werner band systems produce numerous absorption features in the spectra. The $\mathrm{H}_{2}$ optical depth templates developed by McCandliss (2003) for the FUSE mission were used in this study to make a best estimate of the $\mathrm{H}_{2}$ attenuation, $\tau_{\mathrm{H}_{2}}$, along each line of sight. Savage et al. (1977) presented measurements from the Copernicus spacecraft of the column densities of interstellar $\mathrm{H}_{2}$ in the $J=0$ and $J=1$ rotational levels of the ground vibrational state $\left(v^{\prime \prime}=0\right), N(0)$ and $N(1)$ respectively. Several of the lines of sight in this study have measurements of $N(0)$ and $N(1)$ detailed in Savage et al. (1977). For those lines of sight without readily available $\mathrm{H}_{2}$ measurements, values for $N(0)$ and $N(1)$ were estimated from $E(B-V)$.

Bohlin et al. (1978) first derived the dust to gas ratio

$$
N\left(\mathrm{H}_{\text {tot }}\right)=\left(5.8 \times 10^{21} \mathrm{~cm}^{-2} \mathrm{mag}^{-1}\right) \mathrm{E}(\mathrm{B}-\mathrm{V}),
$$

where $N\left(\mathrm{H}_{\text {tot }}\right)=\mathrm{N}(\mathrm{H} \mathrm{I})+2 \mathrm{~N}\left(\mathrm{H}_{2}\right)$, from Copernicus data. This relationship (equation [1) was later confirmed by Rachford et al. (2002) using FUSE data. Additionally, Diplas \& Savage (1994) used Ly $\alpha$ absorption measurements from $I U E$ data to derived the ratio

$$
N(\mathrm{H} \mathrm{I})=\left(4.93 \pm 0.28 \times 10^{21} \mathrm{~cm}^{-2} \mathrm{mag}^{-1}\right) E(B-V) \text {. }
$$

Combining equations (11) and (2), the total column density of molecular hydrogen along a given line of sight can be derived to be

$$
N\left(\mathrm{H}_{2}\right)=\left(4.35 \pm 1.40 \times 10^{20} \mathrm{~cm}^{-2} \mathrm{mag}^{-1}\right) \mathrm{E}(\mathrm{B}-\mathrm{V}) .
$$

In this study it assumed that all of the interstellar $\mathrm{H}_{2}$ are in either the $J=0$ or $J=1$ rotational levels, therefore $N\left(\mathrm{H}_{2}\right)=\mathrm{N}(0)+\mathrm{N}(1)$. Savage et al. (1977) found an average value of the kinetic temperature of the gas to be $T_{01}=77 \pm 17 \mathrm{~K}$ toward stars with $N(0)$ and $N(1)$ larger than $10^{18} \mathrm{~cm}^{-2}$. For the 11 lines of sight in this study with Copernicus measurements, all except $\tau$ Sco exhibited $N(0)$ and $N(1)>10^{18} \mathrm{~cm}^{-2}$. Therefore, it assumed that $T_{01}=77 \pm 17 \mathrm{~K}$, from which values for $N(0)$ and $N(1)$ can be derived using the Boltzmann relation and a value for $E(B-V)$.

With values for $N(0)$ and $N(1)$ for each line of sight estimated, the final item needed to produce an estimate $\tau_{\mathrm{H}_{2}}$ from the McCandliss (2003) templates is an estimate of the Doppler parameter, $b$. Spitzer et al. (1974) provided measurements of $b$ toward four stars in this study. As shown in Savage et al. (1977), with values for $N(0) \sim 10^{19} \mathrm{~cm}^{-2}$, the use of estimated values of $b<10$ $\mathrm{km} \mathrm{s}^{-1}$ should result in small $(\leq 1 \%)$ errors in the overall 
$\mathrm{H}_{2}$ profile. Therefore, an average value of $b=5 \mathrm{~km} \mathrm{~s}^{-1}$ was assumed toward each star, except for $\tau$ Sco where $N(0) \leq 1.66 \times 10^{13} \mathrm{~cm}^{-2}$, in which case the reported value of $b=3.8 \mathrm{~km} \mathrm{~s}^{-1}$ was used. Each of derived $\tau_{\mathrm{H}_{2}}$ templates were smoothed to the SPINR spectral resolution $(\sim 10 \AA)$ then used in determining the total optical depth toward each star as described in section 3.2.3.

\subsection{3. $R_{V}$ Parameter and Optical Depths}

In this study, the ratio of total to selective extinction, $R_{V}=A(V) / E(B-V)$, for each line of sight has been derived from available IR data according to the method presented in Fitzpatrick (1999). The majority of the IR data used in this study came from the Two Micron All Sky Survey (2MASS) database (Skrutskie et al. 2006). However, the brightest stars in the SPINR FOV saturated the 2MASS instrument, so alternative IR measurements were sought from various sources. For $\delta$ Sco (HD 143275), $\sigma$ Sco (HD 147165), $\rho$ Oph (HD 147933), and $\chi \mathrm{Oph}, \mathrm{J}(1.25 \mu \mathrm{m}), \mathrm{H}(1.65 \mu \mathrm{m}), \mathrm{K}(2.2 \mu \mathrm{m})$ band data were taken from Thé et al. (1986). Additionally, IR data from Leitherer \& Wolf (1984), Carter (1990), and Johnson (1966) were used for determining $R_{V}$ along the lines of sight to $\beta$ Sco, $\omega$ Sco, and $\nu$ Sco respectively.

Values for $E(V-J), E(V-H)$, and $E(V-K)$ were then calculated using the intrinsic color indices from Wegner (1994) corresponding to the MK classifications presented in Table 1. For lines of sight with $E(B-V)<0.1, R_{V}=3.1$ was assumed since these small values would generally lead to large discrepancies in the calculation of $R_{V}$. Because $\alpha$ Sco B (HD 148479) is the companion star to an IR bright $M$ type star (HD 148478), it proved difficult to use IR data to predict the value of $R_{V}$ in the $\alpha$ Sco region, therefore the $R_{V}$ value of its nearest neighbor (HD 148605) was used. The derived values for $R_{V}$ are presented in Table 5 .

The optical depth as a function of wavelength, $\tau(\lambda)$, for each line of sight was calculated by

$$
\tau(\lambda)=\frac{E(B-V)}{1.086}\left[k(\lambda-V)+R_{V}\right]+\tau_{\mathrm{H}_{2}}(\lambda),
$$

using the derived extinction curves $(k(\lambda-V)), \mathrm{H}_{2}$ attenuations $\left(\tau_{\mathrm{H}_{2}}(\lambda)\right)$, and $R_{V}$ parameters. With $\tau(\lambda)$ determined, the dereddened luminosity of each star in the FOV is simply calculated by

$$
L_{0}(\lambda)=L(\lambda) e^{\tau(\lambda)},
$$

where $L_{0}$ represents the dereddened luminosity and $L$ represents the reddened luminosity. The $L_{0}$ spectra were then summed over the appropriate wavelength bands (Table 6).

The effective optical depth, $\tau_{e f f}$, was calculated using the weighted average over each of the SPINR wavelength bands given by

$$
\tau_{e f f}=\frac{\sum_{\lambda_{1}}^{\lambda_{2}} \tau(\lambda) L(\lambda)}{\sum_{\lambda_{1}}^{\lambda_{2}} L(\lambda)}
$$

where $L(\lambda)$ includes SPINR's sensitivity function. Overall, the contributions of $\mathrm{H}_{2}$ absorption, $\tau_{\mathrm{H}_{2}}$, to $\tau_{\text {eff }}$ in each wavelength band are small compared to extinction by dust $[\sim 0 \%$ in the $1235-1450 \AA$ band, $\sim 6 \%$ in 1030 $1200 \AA$ band, and $\sim 14 \%$ in the $912-1029 \AA$ band].

\subsection{Nebular Flux}

The total nebular flux was determined by taking an average over the detector pixels that were not known to be part of a stellar spectrum. Due to the nature of the experiment's geometry these nebular pixels are not specific to any one portion to the Upper Scorpius Cloud, but instead probe the nebulosity over the entire SPINR FOV. The radiative transfer model assumes a uniform sphere of dust, which best measures the average nebulosity in the Scorpius region as constrained by the average nebular flux. Figure 2 shows the unprocessed spectrum extracted for the nebular flux. An estimate of the background was determined from the mean of the spectrum from $750-800 \AA$ which should be essentially equal to zero. It is easy to see that this spectrum is contaminated with telluric emission and may contain emission from other sources besides dust such as $\mathrm{H}_{2}$ fluorescence. The most prominent telluric lines in the nebular spectrum are from O II at $834 \AA$, a collection of higher lyman lines and lyman continuum from 906 to $918 \AA$, H I at $972 \AA$ (Ly $\gamma)$, $1025 \AA(\mathrm{Ly} \beta)$, and $1216 \AA(\mathrm{Ly} \alpha)$, and $\mathrm{O} \mathrm{I}$ at $1304 \AA$ (Chakrabarti et al. 1984). These telluric emissions were corrected for by determining the continuum level under each feature and removing the excess flux in those regions. The asymmetry in the Ly $\alpha$ feature is the result of the wings of the Ly $\alpha$ line impinging on the higher sensitivity areas of the detector (Paper I). Figure 2 presents the Ly $\alpha$ line spread function (LSF) scaled to account for this asymmetry. This scaled LSF was used to determine the appropriate amount of flux to attribute to Ly $\alpha$ in the 1235-1450 A bandpass.

Determining how much, if any, $\mathrm{H}_{2}$ fluorescence was contributing to the nebular flux involved producing a $\mathrm{H}_{2}$ emission model that best fit the data as described in section 3.3.1. The final value of the nebular flux, $F_{N}$, for each bandpass was determined by

$$
F_{N}=\sum_{\lambda_{1}}^{\lambda_{2}} I_{N}(\lambda)-\sum_{\lambda_{1}}^{\lambda_{2}} I_{H_{2}}(\lambda)-\sum_{\lambda_{1}}^{\lambda_{2}} I_{\text {telluric }}(\lambda),
$$

where $I_{N}(\lambda)$ represents the unprocessed nebular spectrum, $I_{\mathrm{H}_{2}}(\lambda)$ is the $\mathrm{H}_{2}$ fluorescence model, and $I_{\text {telluric }}(\lambda)$ represents identified telluric emission. Additionally, a small correction (less than 5\%) was subtracted from the nebular flux to account for any residual starlight that may have been lumped in with the nebular spectrum. Uncertainties in the determination of $I_{\text {telluric }}(\lambda)$ and $I_{\mathrm{H}_{2}}(\lambda)$ were propagated to determine the overall uncertainty in $F_{N}$ and hence $F_{N} / F_{*}$ (Table 2). Figure 3 presents the processed nebular spectrum, which has been scaled to match the appropriate detector area.

\subsection{1. $\mathrm{H}_{2}$ Emission}

Molecular hydrogen $\left(\mathrm{H}_{2}\right)$ is the most abundant molecule in the ISM, with dipole-allowed band systems that span $\sim 912-1650 \AA$. These are readily observed as a series of strong absorption features in the 912-1110 $\AA$ spectra of hot stars (as discussed in section 3.2.2). The re-emission that follows as the molecules cascade back to excited rovibrational levels of the ground electronic state produces a highly structured spectrum that has been observed in the diffuse ISM (Martin et al. 
1990; Lee et al. 2006) and in photodissociation regions near massive stars (Witt et al. 1989; France et al. 2004; France \& McCandliss 2005). Consequently, efforts to determine the grain properties from scattered light observations at FUV wavelengths should account for the contribution, if any, from $\mathrm{H}_{2}$ fluorescence (Witt et al. 1993; Shalima et al. 2006).

This study used a model of $\mathrm{H}_{2}$ fluorescence to assess the importance of these emissions and correct for their contribution to the nebular spectra obtained by the SPINR experiment. The $\mathrm{H}_{2}$ fluorescence model, described in France et al. (2005), was adopted for the present work, which takes input parameters such as the total column density, Doppler $b$-value, and the distribution of molecules among the rovibrational levels of the ground electronic state. The code computes photoexcitation rates into the upper electronic states that depend on the strength of the illuminating radiation field at the transition wavelength. These excited levels decay to the ground electronic state, obeying the appropriate branching ratios and selection rules Abgrall et al. 1993a, b). These transitions produce the synthetic fluorescence spectrum.

The unprocessed nebular spectrum presented in Figure 3 shows the presence of emission features in addition to those attributed to telluric lines, which is an indication of the likely presence of $\mathrm{H}_{2}$ fluorescence in the Upper Scorpius region. However, the FOV and spectral resolution of the SPINR instrument makes a determination of the exact properties of the fluorescent $\mathrm{H}_{2}$ challenging, thus a template spectrum was created that can be scaled to the observed flux level, enabling the subtraction of the flux due to $\mathrm{H}_{2}$ fluorescence from the nebular data. Typical values for the column density of the diffuse $\operatorname{ISM}\left(\mathrm{N}\left(\mathrm{H}_{2}\right)=10^{20} \mathrm{~cm}^{-2}\right)$ and $b$-value $\left(2 \mathrm{~km} \mathrm{~s}^{-1}\right)$ were assumed for the model. The ground state population distribution is uncertain. Noting that several studies of photoexcited $\mathrm{H}_{2}$ emission are characterized by rotational excitation temperatures of order several hundred K (Martini et al. 1999; Habart et al. 2004; Allers et al. 2005; France et al. 2007), A value of $\mathrm{T}\left(\mathrm{H}_{2}\right)=500 \mathrm{~K}$ was adopted. By contrast, observational studies suggesting radiatively excited $\mathrm{H}_{2}$ at the diffuse ISM $\mathrm{H}_{2}$ kinetic temperature of $\sim 75 \mathrm{~K}$ (Savage et al. 1977; Burgh et al. 2007) have not been found in the ISM. It is likely that within the large SPINR FOV cold $(77 \mathrm{~K}) \mathrm{H}_{2}$ populations that are responsible for the absorption of stellar UV light coexist with warm ( $500 \mathrm{~K}) \mathrm{H}_{2}$ populations that are responsible for the observed $\mathrm{H}_{2}$ fluorescence.

The illuminating radiation field was assumed to be a lightly reddened B0IV star, characteristic of the spectral type of the hot stars in Scorpius and the average interstellar radiation field (Draine 1978). This radiation field was created from FUSE spectra of a B0IV star from 916.6-1181.9 $\AA$, covering essentially all of the absorbing transitions for $\mathrm{T}\left(\mathrm{H}_{2}\right)=500 \mathrm{~K}$. Since the template spectrum was scaled, changes in absolute flux were not of concern. Changes in spectral shape caused by varying the temperature and radiation field were largely washed out by convolving the model to the $10 \AA$ instrumental resolution.

In summing over the large SPINR FOV, an ensemble of illuminating radiation fields and $\mathrm{H}_{2}$ column densities were taken, thus no attempt was made to reproduce the absolute flux of the observed emission. The individual rotational lines are not resolved with SPINR, and the long wavelength cut-off (1450 ̊) does not permit a measurement of the 1578/1608 ratio. However, detection of $\mathrm{H}_{2}$ fluorescence and dust scattered light with SPINR allows the determination of their relative contribution to the FUV light from Scorpius. The scaled synthetic $\mathrm{H}_{2}$ fluorescence spectrum and the residual dust scattered light from the SPINR nebular data were integrated over three of the model component bands (912-1029 $\AA, 1030-1200$ $\AA, 1235-1450 \AA)$. The relative contribution of $\mathrm{H}_{2}$ emission to the diffuse FUV light in Scorpius in each of the SPINR bandpasses is defined as

$$
f_{j}^{H_{2}}=\frac{\sum_{\lambda_{1}}^{\lambda_{2}} I_{H_{2}}(\lambda)}{\sum_{\lambda_{1}}^{\lambda_{2}}\left(F_{N}(\lambda)+I_{H_{2}}(\lambda)\right)},
$$

where $j$ is the FUV bandpass, $I_{\mathrm{H}_{2}}(\lambda)$ is the fluorescence model, and $F_{N}(\lambda)$ is the dust scattered light. The relative contribution of $\mathrm{H}_{2}$ fluorescence to the nebular light is $\approx 29 \%$ in all three bands, with individual values of $\left[f_{912-1029}^{\mathrm{H}_{2}}, f_{1030-1200}^{\mathrm{H}_{2}}, f_{1235-1450}^{\mathrm{H}_{2}}\right]=[0.272 \pm 0.069$, $0.314 \pm 0.079,0.284 \pm 0.071]$.

This determination of $f_{j}^{H_{2}}$ is consistent with early theoretical considerations by Jakobsen (1982). Using bandpass parameters similar to those chosen for the SPINR observations, he predicts that $\mathrm{H}_{2}$ fluorescence could account for $\sim 30 \%$ of the total nebular flux $\left(f_{1500}^{H_{2}} \sim 0.3\right)$. This finding is also in agreement with the Berkeley UVX Shuttle Spectrometer observations of the diffuse ISM (Martin et al. 1990), which provided the first direct detection of $\mathrm{H}_{2}$ fluorescence in the diffuse ISM. The SPINR results are in excellent agreement with their Targets $4\left(l, b=168^{\circ},-16^{\circ} ; f_{1550}^{H_{2}}=0.27\right)$ and $6(142,35$; $\left.f_{1550}^{H_{2}}=0.32\right)$, and reasonable agreement with their Targets $2\left(132,40 ; f_{1550}^{H_{2}}=0.33\right)$ and $5\left(135,25 ; f_{1550}^{H_{2}}=0.25\right)$.

\section{RESULTS AND DISCUSSION}

Dust model simulations were run for each of the SPINR wavelength bands to identify the value for the dust albedo, $a$, that produced the best fit to the data. This was accomplished by finding the $a$ value that reproduced the $F_{N} / F_{*}$ for each bandpass while varying the asymmetry parameter, $g$, from 0.0 to 0.99 . As shown in Figure 4, there was very little variation in the best-fit value for the albedo over the entire range of $g$ values for all three of the SPINR bandpasses and the two STS-39 FUVCam bandpasses. The best fit values for the dust albedo were $a=0.28 \pm 0.07$ in the $912-1029 \AA$ bandpass, $a=0.33 \pm 0.07$ in the $1030-1200 \AA$ bandpass, and $a=0.77 \pm 0.13$ in the $1235-1450 \AA$ bandpass. Additionally, the STS-39 data presented in Gordon et al. (1994) were used in the updated radiative transfer model to make new estimates of the dust albedo in the Upper Scorpius region for the STS-39 FUVCam 1230-1600 A16502000 Abandpasses. The new albedo values that provided the best fit to the STS-39 FUVCam data were slightly modified from the original estimate of $a=0.47-0.70$ for $\lambda_{\text {eff }}=1362 \AA$ and $a=0.55-0.72$ for $\lambda_{\text {eff }}=1769 \stackrel{\circ}{A}$, to $a=0.54 \pm 0.06$ and $a=0.59 \pm 0.07$ respectively. 
The optical depth of each star in the model Scorpius cloud is determined mostly from the derived extinction curves, but also has some dependence on the values selected for $R_{V}$ and $\tau_{\mathrm{H}_{2}}$ (see section 3.2.3). In order to test the strength of the dependence of the derived albedo values on $R_{V}$ and $\tau_{\mathrm{H}_{2}}$, simulations were run for models with no $\mathrm{H}_{2}$ absorption and $R_{V}=3.1$ for every stellar line of sight. Not accounting for $\mathrm{H}_{2}$ absorption and using a uniform value of $R_{V}=3.1$ reduced the albedo values by $<5 \%$, which is still well within the quoted uncertainties for $a$. Dust model simulations were also run where presence of $\mathrm{H}_{2}$ fluorescence in the Upper Scorpius cloud was ignored (see section 3.3.1). Not accounting for $\mathrm{H}_{2}$ emission in the nebular data results in a $\sim 40 \%$ increase in $F_{N} / F_{*}$. This increase in $F_{N} / F_{*}$ resulted in values for the albedo of $a=0.35 \pm 0.05$ in the $912-1029 \AA$ bandpass, $a=0.43 \pm 0.05$ in the $1030-1200 \AA$ bandpass, and $a=0.93 \pm 0.04$ in the $1235-1450 \AA$ bandpass, which are all within $2 \sigma$ of the best albedo estimates accounting for $\mathrm{H}_{2}$ emission. Overall, the derived albedo values are not particularly sensitive to the amount of $\mathrm{H}_{2}$ absorption or $R_{V}$ value selected along any given line of sight. However, moderate inconsistencies can be introduced into the albedo values if $\mathrm{H}_{2}$ fluorescence is not considered.

Because SPINR was able to record spatial information about the dust in the Upper Scorpius region in addition to spectral information, it was possible to determine the value of $g$ that best reproduced the SPINR data. Model images were produced over a range of $g$ values from 0.00 to 0.99 and with $a$ values corresponding to the best fit for each $g$ value (Figure 4). These model images were "spun" into sinogram space using a transformation matrix and then compared to the SPINR sinograms using a cross-correlation metric. The error in the crosscorrelation metric was determined using a Monte Carlo simulation in which up to $\pm 1 \sigma$ worth of noise was added to each pixel in the SPINR sinogram. The optimal $g$ value was determined from the image/sinogram with the highest cross-correlation to the SPINR data. The range of acceptable $g$ values was determined from the Monte Carlo error estimate on the highest cross-correlation. All $g$ cross-correlation metrics that fell within the error of the optimal fit were deemed acceptable.

The $g$ value that provided the best fit to the SPINR 1030-1200 $\AA$ bandpass data was $g=0.96_{-0.18}^{+0.02}$. Figure 5 shows sinogram and image data for the SPINR 1030-1200 $\AA$ bandpass, the corresponding best fit dust $\operatorname{model}(g=0.96$ and $a=0.30)$, and a model outside of the range of acceptable $a$ and $g$ values $(g=0.0$ and $a=0.75)$. In the $912-1029 \AA$ and $1235-1450 \AA$ bandpasses no statistically significant constraints could be put on the $g$ value. It is not surprising that values for $g$ are not well determined in this study since the geometry of the nebula in Upper Scorpius was selected for its weak dependence on $g$ to better determine $a$. However, given the constraint from the $1030-1200 \AA$ bandpass we can confidently say that $g>0.42$ at the three sigma level. This is not a tight constraint on $g$, but does align with the general expectation for $g$ to be highly forward scattering, $g>0.7$, as shown by previous predictions for $g$ in the FUV (Gordon 2004).

The results for $a$ from this study are compared with previous studies of reflection nebulae in Figure 6. The values of $a$ derived for the 912-1029 $\AA$ and 1030-1200 $\AA$ bandpasses correspond well with both previous observational determinations $a$ and the Weingartner \& Draine (2001) model in this spectral region. The value of $a$ determined for the 1235-1450 $\AA$ bandpass is on the high end of the observational data points scattered within the 1200$1400 \AA$ range, but are well within $3 \sigma$ of the other observational determination of $a$. However, what is clear from this study and previous observational studies of albedo in the FUV is that the dust models (Weingartner \& Draine 2001; Clayton et al. 2003; Zubko et al. 2004) do not correctly account for the apparent rise in the albedo from around $1200 \AA$ to $1800 \AA$. Further observational studies of the ISM in this spectral region are needed to fully characterize this rise in the albedo and determine its source.

\section{SUMMARY}

Both the stellar and nebular populations of the Upper Scorpius region have been characterized in the FUV (912-1450 ^) in this study. The derived nebular and stellar flux levels were used to constrain a radiative transfer model and make a determination of the $a$ and $g$ values that the dust grains in the region possess for three

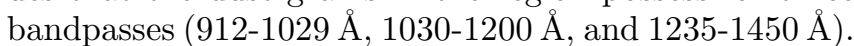
Overall, the values for $a$ and $g$ derived in this study corroborate previous findings that dust in the ISM is highly forward scattering $(g>0.7)$ and possess a low albedo $(a<0.4)$ in the $912-1200 \AA$ wavelength regime.

The authors would like to thank Dr. George S. Carruthers for providing us with the images of the Upper Scorpius region from the STS-39 FUVCam experiment, the Wallops sounding rocket staff for their support, and Ms. Valerie Gsell for directing the payload. We would also like to thank our anonymous referee for their helpful comments and suggestions. This publication makes use of data products from the Two Micron All Sky Survey, which is a joint project of the University of Massachusetts and the Infrared Processing and Analysis Center/California Institute of Technology, funded by the National Aeronautics and Space Administration and the National Science Foundation. This work was supported in part by NASA grant NAG5-690.

\section{APPENDIX}

\section{REFERENCES}

Abgrall, H., Roueff, E., Launay, F., Roncin, J. Y., \& Subtil, J. L. 1993a, A\&AS, 101, 273

-. 1993b, A\&AS, 101, 323

Allers, K. N., Jaffe, D. T., Lacy, J. H., Draine, B. T., \& Richter,

M. J. 2005, ApJ, 630, 368
Bohlin, R. C., Savage, B. D., \& Drake, J. F. 1978, ApJ, 224, 132 Burgh, E. B., France, K., \& McCandliss, S. R. 2007, ApJ, 658, 446 Burgh, E. B., McCandliss, S. R., \& Feldman, P. D. 2002, ApJ, 575,240 
Calzetti, D., Bohlin, R. C., Gordon, K. D., Witt, A. N., \& Bianchi, L. 1995, ApJ, 446, L97+

Carter, B. S. 1990, MNRAS, 242, 1

Chakrabarti, S., Kimble, R., \& Bowyer, S. 1984, J. Geophys. Res., 89, 5660

Clayton, G. C., Wolff, M. J., Sofia, U. J., Gordon, K. D., \& Misselt, K. A. 2003, ApJ, 588, 871

de Geus, E. J., de Zeeuw, P. T., \& Lub, J. 1989, A\&A, 216, 44

de Jager, C. \& Nieuwenhuijzen, H. 1987, A\&A, 177, 217

Diplas, A. \& Savage, B. D. 1994, ApJ, 427, 274

Draine, B. T. 1978, ApJS, 36, 595

-. 2003, ApJ, 598, 1017

Fitzpatrick, E. L. 1999, PASP, 111, 63

Fitzpatrick, E. L. 2004, in Astronomical Society of the Pacific Conference Series, Vol. 309, Astrophysics of Dust, ed. A. N. Witt, G. C. Clayton, \& B. T. Draine, 33-+

Fitzpatrick, E. L. \& Massa, D. 1988, ApJ, 328, 734

France, K., Andersson, B.-G., McCandliss, S. R., \& Feldman, P. D. 2005, ApJ, 628, 750

France, K. \& McCandliss, S. R. 2005, ApJ, 629, 97

France, K., McCandliss, S. R., Burgh, E. B., \& Feldman, P. D. 2004, ApJ, 616, 257

France, K., McCandliss, S. R., \& Lupu, R. E. 2007, ApJ, 655, 920

Gaustad, J. E. \& van Buren, D. 1993, PASP, 105, 1127

Gordon, K. D. 2004, in ASP Conf. Ser. 309: Astrophysics of Dust, ed. A. N. Witt, G. C. Clayton, \& B. T. Draine, 77-91

Gordon, K. D., Misselt, K. A., Witt, A. N., \& Clayton, G. C. 2001, ApJ, 551, 269

Gordon, K. D., Witt, A. N., Carruthers, G. R., Christensen, S. A., \& Dohne, B. C. 1994, ApJ, 432, 641

Green, J. C., Snow, T. P., Cook, T. A., Cash, W. C., \& Poplawski, O. 1992, ApJ, 395, 289

Habart, E., Boulanger, F., Verstraete, L., Walmsley, C. M., \& Pineau des Forêts, G. 2004, A\&A, 414, 531

Hoffleit, D. \& Warren, Jr., W. H. 1991, The Bright Star Catalogue, 5th Revised Ed. (New Haven, Conn.: Yale University Observatory)

Jakobsen, P. 1982, A\&A, 106, 375

Johnson, H. L. 1966, ARA\&A, 4, 193

Kurucz, R. L. 1992, in IAU Symp. 149, The Stellar Populations of Galaxies, ed. B. Barbuy \& A. Renzini (Dordrecht:Kluwer), 225

Lee, D.-H., Yuk, I.-S., Jin, H., Seon, K.-I., Edelstein, J., Korpela, E. J., Adolfo, J., Min, K.-W., Ryu, K.-S., Shinn, J.-H., \& van Dishoeck, E. F. 2006, ApJ, 644, L181

Leitherer, C. \& Wolf, B. 1984, A\&A, 132, 151

Martin, C., Hurwitz, M., \& Bowyer, S. 1990, ApJ, 354, 220

Martini, P., Sellgren, K., \& DePoy, D. 1999, ApJ, 526, 772

Mathis, J. S., Whitney, B. A., \& Wood, K. 2002, ApJ, 574, 812

McCandliss, S. R. 2003, PASP, 115, 651

Misselt, K. A., Gordon, K. D., Clayton, G. C., \& Wolff, M. J. 2001, ApJ, 551, 277
Papaj, J., Krelowski, J., \& Wegner, W. 1991, MNRAS, 252, 403 Piña, R. K. \& Puetter, R. C. 1993, PASP, 105, 630

Rachford, B. L., Snow, T. P., Tumlinson, J., Shull, J. M., Blair, W. P., Ferlet, R., Friedman, S. D., Gry, C., Jenkins, E. B., Morton, D. C., Savage, B. D., Sonnentrucker, P., Vidal-Madjar, A., Welty, D. E., \& York, D. G. 2002, ApJ, 577, 221

Savage, B. D., Bohlin, R. C., Drake, J. F., \& Budich, W. 1977, ApJ, 216, 291

Shalima, P., Sujatha, N. V., Murthy, J., Henry, R. C., \& Sahnow, D. J. 2006, MNRAS, 367, 1686

Skrutskie, M. F., Cutri, R. M., Stiening, R., Weinberg, M. D., Schneider, S., Carpenter, J. M., Beichman, C., Capps, R. Chester, T., Elias, J., Huchra, J., Liebert, J., Lonsdale, C., Monet, D. G., Price, S., Seitzer, P., Jarrett, T., Kirkpatrick, J. D., Gizis, J. E., Howard, E., Evans, T., Fowler, J., Fullmer, L., Hurt, R., Light, R., Kopan, E. L., Marsh, K. A., McCallon, H. L., Tam, R., Van Dyk, S., \& Wheelock, S. 2006, AJ, 131, 1163

Snow, Jr., T. P., Buss, Jr., R. H., Gilra, D. P., \& Swings, J. P. 1987, ApJ, 321, 921

Sofia, U. J., Wolff, M. J., Rachford, B., Gordon, K. D., Clayton,

G. C., Cartledge, S. I. B., Martin, P. G., Draine, B. T., Mathis, J. S., Snow, T. P., \& Whittet, D. C. B. 2005, ApJ, 625, 167

Spitzer, Jr., L., Cochran, W. D., \& Hirshfeld, A. 1974, ApJS, 28, 373

Straižys, V. \& Kuriliene, G. 1981, Ap\&SS, 80, 353

Sujatha, N. V., Murthy, J., Shalima, P., \& Henry, R. C. 2007, ApJ, 665, 363

Sujatha, N. V., Shalima, P., Murthy, J., \& Henry, R. C. 2005, ApJ, 633, 257

Thé, P. S., Wesselius, P. R., \& Janssen, I. M. H. H. 1986, A\&AS, 66,63

Wegner, W. 1994, MNRAS, 270, 229

—. 2003, Astronomische Nachrichten, 324, 219

Weingartner, J. C. \& Draine, B. T. 2001, ApJ, 548, 296

Wesselius, P. R., van Duinen, R. J., de Jonge, A. R. W., Aalders,

J. W. G., Luinge, W., \& Wildeman, K. J. 1982, A\&AS, 49, 427

Witt, A. N., Petersohn, J. K., Holberg, J. B., Murthy, J., Dring, A., \& Henry, R. C. 1993, ApJ, 410, 714

Witt, A. N., Stecher, T. P., Boroson, T. A., \& Bohlin, R. C. 1989, ApJL, 336, L21

Wu, C., Mo, J., Crenshaw, D. M., \& Schiffer, F. H. 1998, in ESA SP-413: Ultraviolet Astrophysics Beyond the IUE Final Archive, ed. W. Wamsteker, R. Gonzalez Riestra, \& B. Harris, $751-+$

Zubko, V., Dwek, E., \& Arendt, R. G. 2004, ApJS, 152, 211 
TABLE 1

Stellar Data

\begin{tabular}{|c|c|c|c|c|c|c|c|c|}
\hline No. & HD & Name & $1\left(^{\circ}\right)$ & $\mathrm{b}\left({ }^{\circ}\right)$ & $\mathrm{MK}^{\mathrm{a}}$ & $\mathrm{V}_{\text {mag }}{ }^{\mathrm{a}}$ & $\mathrm{E}(\mathrm{B}-\mathrm{V})^{\mathrm{b}}$ & IUE Data \\
\hline 1 & 149438 & $\tau$ Sco & 351.535 & 12.807 & B0V & 2.82 & 0.034 & swp33008 \\
\hline 2 & 143275 & $\delta$ Sco & 350.096 & 22.491 & B0.3IV & 2.32 & 0.120 & \\
\hline 3 & 144217 & $\beta$ Sco & 353.197 & 23.601 & $\mathrm{~B} 1 \mathrm{~V}$ & 2.62 & 0.170 & $\ldots$ \\
\hline 4 & 143018 & $\pi$ Sco & 347.214 & 20.231 & $\mathrm{~B} 1 \mathrm{~V}$ & 2.89 & 0.065 & $\ldots$ \\
\hline 5 & 147165 & $\sigma \mathrm{Sco}$ & 351.314 & 16.998 & B1III & 2.89 & 0.340 & \\
\hline 6 & 142669 & $\rho$ Sco & 344.628 & 18.272 & B2IV-V & 3.88 & 0.022 & swp32877 \\
\hline 7 & 144470 & $\omega$ Sco & 352.751 & 22.772 & B1V & 3.96 & 0.190 & $\ldots$ \\
\hline 8 & 145502 & $\nu \mathrm{Sco}$ & 354.608 & 22.700 & B3V & 4.01 & 0.250 & $\ldots$ \\
\hline 9 & 145482 & $13 \mathrm{Sco}$ & 348.117 & 16.836 & $\mathrm{~B} 2 \mathrm{~V}$ & 4.59 & 0.044 & \\
\hline 10 & 148605 & 22 Sco & 353.099 & 15.796 & $\mathrm{~B} 2 \mathrm{~V}$ & 4.79 & 0.069 & swp09221\&lwr07977 \\
\hline 11 & 141637 & $1 \mathrm{Sco}$ & 346.098 & 21.705 & B3V & 4.64 & 0.160 & swp42216\&lwp20988 \\
\hline 12 & 142114 & $2 \mathrm{Sco}$ & 346.876 & 21.614 & $\mathrm{~B} 2.5 \mathrm{Vn}$ & 4.59 & 0.140 & swp20611\&lwr16524 \\
\hline 13 & 147933 & $\rho \mathrm{Oph}$ & 353.687 & 17.687 & B2IV & 4.63 & 0.450 & \\
\hline 14 & $148479^{\mathrm{c}}$ & $\alpha$ Sco B & 351.946 & 15.065 & B3V & 4.91 & 0.150 & swp05892\&lwr05146 \\
\hline 15 & 148184 & $\chi \mathrm{Oph}$ & 357.932 & 20.677 & B2IVpe & 4.42 & 0.490 & swp54090\&lwp30205 \\
\hline 16 & 142096 & $\widehat{\lambda} \mathrm{Lib}$ & 350.725 & 25.378 & $\mathrm{~B} 2.5 \mathrm{~V}$ & 5.03 & 0.200 & swp42326\&lwr10778 \\
\hline 17 & 142184 & $\ldots$ & 347.932 & 22.545 & B2.5Vne & 5.42 & 0.155 & swp36741\&lwp15993 \\
\hline 18 & 142990 & $\ldots$ & 348.121 & 21.196 & B5IV & 5.43 & 0.090 & swp 42227 \\
\hline 19 & 142165 & & 347.514 & 22.149 & B6IVn & 5.39 & 0.113 & swp42217\&lwp17717 \\
\hline 20 & 142378 & $47 \mathrm{Lib}$ & 351.648 & 25.657 & $\mathrm{~B} 5 \mathrm{~V}$ & 5.94 & 0.140 & swp09236\&lwr07996 \\
\hline 21 & 142883 & $\ldots$ & 350.885 & 24.085 & B3V & 5.85 & 0.200 & swp36751\&lwp16004 \\
\hline 22 & 144334 & & 350.348 & 20.855 & B8p & 5.92 & 0.081 & swp21083\&lwr16819 \\
\hline 23 & 142301 & $3 \mathrm{Sco}$ & 347.124 & 21.513 & BB8IIIp & 5.87 & 0.110 & swp21092\&lwr16825 \\
\hline 24 & 142250 & - & 345.568 & 20.005 & $\mathrm{~B} 6 \mathrm{Vp}$ & 6.14 & 0.050 & swp36742 \\
\hline 25 & 146001 & $\ldots$ & 350.386 & 18.119 & B7IV & 6.05 & 0.153 & swp38567\&lwp16003 \\
\hline 26 & 145792 & $\ldots$ & 351.011 & 19.029 & $\mathrm{~B} 5 \mathrm{~V}$ & 6.41 & 0.169 & swp36749\&lwp16002 \\
\hline 27 & 144661 & & 349.992 & 19.970 & B7IIIp & 6.33 & 0.097 & swp13953 \\
\hline 28 & 145483 & $12 \mathrm{Sco}$ & 347.745 & 16.499 & B9V & 5.67 & 0.059 & swp16306 \\
\hline 29 & 144844 & $\ldots$ & 350.734 & 20.369 & B9IVp & 5.88 & 0.116 & swp36828 \\
\hline 30 & 142884 & $\ldots$ & 348.963 & 22.255 & B9p & 6.79 & 0.159 & swp36743 \\
\hline 31 & 146416 & $\ldots$ & 353.981 & 20.596 & B9V & 6.61 & 0.075 & swp36831 \\
\hline 32 & 142315 & $\ldots$ & 348.980 & 23.299 & $\mathrm{~B} 8 \mathrm{~V}$ & 6.86 & 0.116 & swp36837\&lwp16118 \\
\hline 33 & 145102 & & 348.546 & 17.872 & B9Vp & 6.59 & 0.144 & swp36829\&lwp16110 \\
\hline \multicolumn{9}{|c|}{ Additional Standard Stars ${ }^{\mathrm{d}}$} \\
\hline & 122980 & $\chi \mathrm{Cen}$ & 317.730 & 19.538 & $\mathrm{~B} 2 \mathrm{~V}$ & 4.35 & 0.04 & swp46857\&lwp24818 \\
\hline$\ldots$ & 32630 & $\eta$ Aur & 165.354 & 00.272 & B3V & 3.17 & 0.02 & swp08197\&lwr07125 \\
\hline$\ldots$ & 3360 & $\zeta$ Cas & 120.776 & -08.914 & B2IV & 3.66 & 0.04 & swp04316\&lwr03812 \\
\hline$\ldots$ & 61831 & & 252.138 & -07.898 & $\mathrm{~B} 2.5 \mathrm{~V}$ & 4.84 & 0.03 & swp14309\&lwr10940 \\
\hline$\ldots$ & 90994 & $\beta$ Sex & 246.415 & 46.167 & $\mathrm{~B} 6 \mathrm{~V}$ & 5.07 & 0.00 & swp15791\&lwr12162 \\
\hline & 34759 & $\rho$ Aur & 166.565 & 02.927 & $\mathrm{~B} 5 \mathrm{~V}$ & 5.22 & 0.01 & swp15537\&lwr09868 \\
\hline$\ldots$ & 29335 & 49 Eri & 195.134 & -28.939 & $\mathrm{~B} 7 \mathrm{~V}$ & 5.31 & 0.00 & swp15788\&lwr12159 \\
\hline & 222173 & $\kappa$ And & 109.766 & -16.714 & B9IVn & 4.14 & 0.07 & swp33853\&lwp13557 \\
\hline$\ldots$ & 196867 & $\alpha$ Del & 060.304 & -15.321 & B9V & 3.77 & 0.01 & swp15545\&lwr12025 \\
\hline
\end{tabular}

${ }^{a}$ From Hoffleit \& Warren (1991).

b $\mathrm{E}(\mathrm{B}-\mathrm{V})$ values taken preferentally from Papaj et al. (1991) then de Geus et al. (1989) and finally Wegner (2003).

${ }^{c}$ MK, $V_{\text {mag }}$, and E(B-V) for HD 148479 were taken from Snow et al. (1987).

d All standard star data taken from Wu et al. (1998). 
TABLE 2

Nebular to Stellar Flux Ratios

\begin{tabular}{crc}
\hline \hline Bandpass & $\lambda_{\text {eff }}$ & $F_{N} / F_{*}$ \\
\hline $912 \AA-1029 \AA$ & $973 \AA$ & $0.377 \pm 0.041$ \\
$1030 \AA-1200 \AA$ & $1106 \AA$ & $0.406 \pm 0.052$ \\
$1235 \AA-1450 \AA$ & $1261 \AA$ & $1.301 \pm 0.159$ \\
\hline
\end{tabular}


TABLE 3

Measured Reddened Luminosities

\begin{tabular}{|c|c|c|c|}
\hline HD & $\begin{array}{c}L[973 \AA] \\
\left(\text { counts s }^{-1} \text { bandpass }^{-1} \text { ) }\right.\end{array}$ & $\begin{array}{c}L[1106 \AA] \\
\text { (counts s }{ }^{-1} \text { bandpass }^{-1} \text { ) }\end{array}$ & $\begin{array}{c}L[1261 \AA] \\
\left(\text { counts s }{ }^{-1} \text { bandpass }^{-1} \text { ) }\right.\end{array}$ \\
\hline 149438 & $6461.77 \pm 42.01$ & $7068.90 \pm 39.63$ & $3620.92 \pm 715.02$ \\
\hline 143275 & $4592.08 \pm 26.21$ & $7523.04 \pm 32.92$ & $3360.57 \pm 27.56$ \\
\hline 144217 & $2319.94 \pm 21.79$ & $4691.78 \pm 29.39$ & $2511.58 \pm 29.78$ \\
\hline 143018 & $3929.67 \pm 26.16$ & $6605.39 \pm 33.14$ & $2449.16 \pm 27.73$ \\
\hline 147165 & $739.23 \pm 15.18$ & $1890.19 \pm 20.74$ & $920.45 \pm 21.66$ \\
\hline 142669 & $798.11 \pm 18.98$ & $1177.58 \pm 20.16$ & $670.29 \pm 132.36$ \\
\hline 144470 & $562.69 \pm 14.21$ & $1325.87 \pm 18.46$ & $783.63 \pm 21.72$ \\
\hline 145502 & $215.81 \pm 12.88$ & $537.18 \pm 15.70$ & $230.42 \pm 22.33$ \\
\hline 145482 & $380.29 \pm 12.47$ & $876.03 \pm 15.59$ & $337.77 \pm 19.20$ \\
\hline 148605 & $306.32 \pm 13.00$ & $687.86 \pm 15.92$ & $316.78 \pm 20.62$ \\
\hline 141637 & $214.14 \pm 12.92$ & $492.26 \pm 15.47$ & $264.32 \pm 25.16$ \\
\hline 142114 & $338.71 \pm 12.42$ & $882.97 \pm 15.85$ & $501.40 \pm 20.30$ \\
\hline 147933 & $158.80 \pm 11.63$ & $323.28 \pm 13.49$ & $221.25 \pm 18.38$ \\
\hline 148479 & $99.14 \pm 11.41$ & $320.55 \pm 13.66$ & $172.12 \pm 20.34$ \\
\hline 148184 & $36.29 \pm 15.99$ & $146.84 \pm 50.20$ & $81.03 \pm 16.00$ \\
\hline 142096 & $44.85 \pm 23.61$ & $146.73 \pm 53.54$ & $89.88 \pm 17.75$ \\
\hline 142184 & $57.28 \pm 31.71$ & $159.24 \pm 59.38$ & $50.87 \pm 10.05$ \\
\hline 142990 & $20.92 \pm 9.80$ & $80.90 \pm 29.08$ & $50.46 \pm 9.96$ \\
\hline 142165 & $17.64 \pm 8.72$ & $70.64 \pm 24.39$ & $29.73 \pm 5.87$ \\
\hline 142378 & $10.75 \pm 4.89$ & $44.85 \pm 14.54$ & $29.88 \pm 5.90$ \\
\hline 142883 & $18.61 \pm 9.45$ & $62.58 \pm 22.31$ & $24.12 \pm 4.76$ \\
\hline 144334 & $0.06 \pm 0.03$ & $2.42 \pm 0.87$ & $21.60 \pm 4.26$ \\
\hline 142301 & $0.06 \pm 0.03$ & $2.34 \pm 0.78$ & $20.93 \pm 4.13$ \\
\hline 142250 & $4.56 \pm 2.13$ & $23.99 \pm 8.62$ & $16.86 \pm 3.33$ \\
\hline 146001 & $1.03 \pm 0.51$ & $8.85 \pm 3.08$ & $12.94 \pm 2.56$ \\
\hline 145792 & $1.83 \pm 1.01$ & $11.93 \pm 4.70$ & $12.71 \pm 2.51$ \\
\hline 144661 & $0.35 \pm 0.16$ & $4.40 \pm 1.58$ & $12.64 \pm 2.50$ \\
\hline 145483 & $0.00 \pm 0.00$ & $0.63 \pm 0.22$ & $8.01 \pm 1.58$ \\
\hline 144844 & $0.04 \pm 0.02$ & $1.87 \pm 0.69$ & $10.71 \pm 2.11$ \\
\hline 142884 & $0.00 \pm 0.00$ & $0.32 \pm 0.11$ & $4.67 \pm 0.92$ \\
\hline 146416 & $0.00 \pm 0.00$ & $0.23 \pm 0.08$ & $3.33 \pm 0.66$ \\
\hline 142315 & $0.03 \pm 0.02$ & $1.14 \pm 0.41$ & $3.45 \pm 0.68$ \\
\hline 145102 & $0.00 \pm 0.00$ & $0.19 \pm 0.07$ & $2.40 \pm 0.47$ \\
\hline
\end{tabular}


TABLE 4

FM88 Curve Coefficients

\begin{tabular}{|c|c|c|c|c|c|c|c|}
\hline HD & Standard(HD) & $c_{1}$ & $c_{2}$ & $c_{3}$ & $c_{4}$ & $\gamma$ & $\lambda_{0}^{-1}$ \\
\hline 149438 & & $-0.07 \pm 0.02$ & $0.70 \pm 0.01$ & $3.23 \pm 0.05$ & $0.41 \pm 0.01$ & $0.99 \pm 0.01$ & $4.596 \pm 0.011$ \\
\hline 143275 & 143018 & $-1.29 \pm 0.22$ & $0.86 \pm 0.04$ & $2.59 \pm 0.35$ & $0.22 \pm 0.03$ & $0.77 \pm 0.06$ & $4.555 \pm 0.014$ \\
\hline 144217 & 143018 & $1.07 \pm 0.15$ & $0.38 \pm 0.03$ & $2.73 \pm 0.19$ & $0.32 \pm 0.02$ & $0.66 \pm 0.03$ & $4.502 \pm 0.007$ \\
\hline 143018 & & $-0.07 \pm 0.02$ & $0.70 \pm 0.01$ & $3.23 \pm 0.05$ & $0.41 \pm 0.01$ & $0.99 \pm 0.01$ & $4.596 \pm 0.011$ \\
\hline 147165 & 143018 & $1.46 \pm 0.08$ & $0.29 \pm 0.01$ & $2.77 \pm 0.14$ & $0.15 \pm 0.01$ & $0.83 \pm 0.02$ & $4.572 \pm 0.006$ \\
\hline 142669 & & $-0.07 \pm 0.02$ & $0.70 \pm 0.01$ & $3.23 \pm 0.05$ & $0.41 \pm 0.01$ & $0.99 \pm 0.01$ & $4.596 \pm 0.011$ \\
\hline 144470 & 143018 & $0.10 \pm 0.15$ & $0.65 \pm 0.03$ & $3.43 \pm 0.27$ & $0.12 \pm 0.03$ & $0.81 \pm 0.03$ & $4.536 \pm 0.008$ \\
\hline 145502 & 143018 & $1.28 \pm 0.12$ & $0.50 \pm 0.02$ & $3.58 \pm 0.26$ & $0.34 \pm 0.03$ & $0.90 \pm 0.03$ & $4.526 \pm 0.008$ \\
\hline 145482 & . & $-0.07 \pm 0.02$ & $0.70 \pm 0.01$ & $3.23 \pm 0.05$ & $0.41 \pm 0.01$ & $0.99 \pm 0.01$ & $4.596 \pm 0.011$ \\
\hline 148605 & & $-0.07 \pm 0.02$ & $0.70 \pm 0.01$ & $3.23 \pm 0.05$ & $0.41 \pm 0.01$ & $0.99 \pm 0.01$ & $4.596 \pm 0.011$ \\
\hline 141637 & 148605 & $-1.29 \pm 0.17$ & $0.73 \pm 0.03$ & $2.11 \pm 0.27$ & $0.17 \pm 0.08$ & $0.77 \pm 0.05$ & $4.554 \pm 0.013$ \\
\hline 142114 & 122980 & $-0.54 \pm 0.20$ & $0.64 \pm 0.04$ & $4.05 \pm 0.43$ & $0.30 \pm 0.10$ & $0.89 \pm 0.05$ & $4.590 \pm 0.012$ \\
\hline 147933 & 143018 & $0.89 \pm 0.06$ & $0.40 \pm 0.01$ & & & & $4.529 \pm 0.004$ \\
\hline 148479 & 32630 & $2.40 \pm 0.20$ & $0.22 \pm 0.01$ & $2.88 \pm 0.37$ & $0.68 \pm 0.09$ & $0.83 \pm 0.06$ & $4.567 \pm 0.013$ \\
\hline 148184 & 3360 & $-0.35 \pm 0.06$ & $0.51 \pm 0.01$ & $3.32 \pm 0.11$ & $0.13 \pm 0$ & $0.84 \pm 0.01$ & $4.538 \pm 0.003$ \\
\hline 142096 & 122980 & $1.22 \pm 0.17$ & $0.53 \pm 0.03$ & $4.11 \pm 0.51$ & $0.34 \pm 0.07$ & $1.08 \pm 0.06$ & $4.586 \pm 0.014$ \\
\hline 142184 & 61831 & $1.42 \pm 0.17$ & $0.50 \pm 0.03$ & $1.38 \pm 0.19$ & 0.09 & $0.63 \pm 0.05$ & $4.581 \pm 0.012$ \\
\hline 142990 & & $-0.07 \pm 0.02$ & & $3 \pm 0.05$ & $0.41 \pm$ & 0.01 & $96 \pm 0.011$ \\
\hline 142165 & 90994 & $0.99 \pm 0.25$ & $0.43 \pm 0.05$ & $2.45 \pm 0.45$ & $0.03 \pm 0.11$ & $0.82 \pm 0.08$ & $4.583 \pm 0.019$ \\
\hline 142378 & 34759 & & & \pm 0.55 & & $9 \pm 0.06$ & $4.532 \pm 0.013$ \\
\hline 142883 & 32630 & $0.65 \pm 0.14$ & $0.58 \pm 0.03$ & $2.14 \pm 0.21$ & $0.28 \pm 0.07$ & $0.73 \pm 0.04$ & $4.560 \pm 0.009$ \\
\hline 144334 & & $-0.07 \pm 0.02$ & $0.70 \pm 0.01$ & $3.23 \pm 0.05$ & $0.41 \pm 0.01$ & $0.99 \pm 0.01$ & $4.596 \pm 0.011$ \\
\hline 142301 & 144334 & $0.35 \pm 0.25$ & $0.56 \pm 0$. & $2.80 \pm 0.51$ & \pm 0.12 & $0.85 \pm 0.08$ & $4.601 \pm 0.019$ \\
\hline 142250 & & $-0.07 \pm 0.02$ & $0.70 \pm 0.01$ & $3.23 \pm 0.05$ & $0.41 \pm 0.01$ & $0.99 \pm 0.01$ & $4.596 \pm 0.011$ \\
\hline 146001 & 29335 & $-0.02 \pm 0.21$ & $0.56 \pm 0.04$ & $4.11 \pm 0.59$ & $0.07 \pm 0.09$ & $1.04 \pm 0.07$ & $4.534 \pm 0.016$ \\
\hline 145792 & 34759 & $1.37 \pm 0.19$ & $0.33 \pm 0.03$ & $3.15 \pm 0.40$ & $0.56 \pm 0.08$ & $0.92 \pm 0.06$ & $4.507 \pm 0.013$ \\
\hline 144661 & & $-0.07 \pm 0.02$ & & $3.23 \pm 0.05$ & & $0.99 \pm 0.01$ & $4.596 \pm 0.011$ \\
\hline 145483 & & $-0.07 \pm 0.02$ & $0.70 \pm 0$ & $3.23 \pm 0.05$ & $0.41 \pm 0.01$ & $0.99 \pm 0.01$ & $4.596 \pm 0.011$ \\
\hline 144844 & $144334^{\mathrm{a}}$ & $-0.07 \pm 0.02$ & $0.70 \pm 0$. & & & & $4.596 \pm 0.011$ \\
\hline & $142184^{\mathrm{a}}$ & & & & & $0.63 \pm 0.05$ & $4.581 \pm 0.012$ \\
\hline 146416 & & $-0.07 \pm 0.02$ & $0.70 \pm 0.01$ & $3.23 \pm 0.05$ & $0.41 \pm 0.01$ & $0.99 \pm 0.01$ & $4.596 \pm 0.011$ \\
\hline & & & & & & $0.85 \pm 0.07$ & \\
\hline 145102 & 196867 & $-1.16 \pm 0.21$ & $0.70 \pm 0.04$ & $3.08 \pm 0.44$ & $0.17 \pm 0.10$ & $0.91 \pm 0.07$ & $4.509 \pm 0.015$ \\
\hline
\end{tabular}

${ }^{a}$ Derived from nearest neighbor 
TABLE 5

$R_{V}$ Values and Stellar Optical Depths

\begin{tabular}{ccccc}
\hline \hline HD & $R_{V}$ & $\tau_{\text {eff }}[973 \AA]$ & $\tau_{\text {eff }}[1106 \AA]$ & $\tau_{\text {eff }}[1261 \AA]$ \\
\hline 149438 & $3.10 \pm 0.10$ & $0.515 \pm 0.332$ & $0.406 \pm 0.263$ & $0.296 \pm 0.191$ \\
143275 & $2.96 \pm 0.48$ & $1.713 \pm 0.293$ & $1.340 \pm 0.254$ & $0.978 \pm 0.188$ \\
144217 & $2.59 \pm 0.26$ & $2.224 \pm 0.259$ & $1.640 \pm 0.217$ & $1.161 \pm 0.157$ \\
143018 & $3.10 \pm 0.10$ & $1.152 \pm 0.339$ & $0.847 \pm 0.286$ & $0.567 \pm 0.192$ \\
147165 & $3.53 \pm 0.19$ & $3.463 \pm 0.216$ & $2.896 \pm 0.197$ & $2.393 \pm 0.166$ \\
142669 & $3.10 \pm 0.10$ & $0.455 \pm 0.342$ & $0.320 \pm 0.320$ & $0.192 \pm 0.192$ \\
144470 & $3.23 \pm 0.24$ & $2.440 \pm 0.254$ & $1.942 \pm 0.230$ & $1.531 \pm 0.183$ \\
145502 & $3.28 \pm 0.23$ & $3.636 \pm 0.306$ & $2.882 \pm 0.261$ & $2.109 \pm 0.194$ \\
145482 & $3.10 \pm 0.10$ & $0.827 \pm 0.339$ & $0.607 \pm 0.303$ & $0.384 \pm 0.192$ \\
148605 & $3.10 \pm 0.10$ & $1.114 \pm 0.325$ & $0.858 \pm 0.273$ & $0.590 \pm 0.188$ \\
141637 & $2.24 \pm 0.34$ & $1.755 \pm 0.310$ & $1.404 \pm 0.207$ & $1.017 \pm 0.150$ \\
142114 & $2.51 \pm 0.29$ & $1.962 \pm 0.413$ & $1.429 \pm 0.238$ & $1.012 \pm 0.165$ \\
147933 & $4.07 \pm 0.15$ & $5.404 \pm 0.249$ & $4.301 \pm 0.220$ & $3.485 \pm 0.182$ \\
148479 & $3.10 \pm 0.10$ & $2.523 \pm 0.433$ & $1.881 \pm 0.281$ & $1.169 \pm 0.172$ \\
148184 & $4.23 \pm 0.14$ & $5.566 \pm 0.251$ & $4.532 \pm 0.215$ & $3.703 \pm 0.178$ \\
142096 & $3.34 \pm 0.25$ & $3.041 \pm 0.375$ & $2.366 \pm 0.269$ & $1.758 \pm 0.200$ \\
142184 & $3.91 \pm 0.38$ & $2.388 \pm 0.408$ & $1.878 \pm 0.278$ & $1.410 \pm 0.208$ \\
142990 & $3.10 \pm 0.10$ & $1.536 \pm 0.328$ & $1.152 \pm 0.281$ & $0.781 \pm 0.191$ \\
142165 & $4.11 \pm 0.51$ & $1.305 \pm 0.375$ & $1.078 \pm 0.228$ & $0.892 \pm 0.183$ \\
142378 & $3.98 \pm 0.40$ & $1.377 \pm 0.315$ & $1.110 \pm 0.192$ & $0.899 \pm 0.152$ \\
142883 & $3.30 \pm 0.25$ & $2.861 \pm 0.358$ & $2.246 \pm 0.256$ & $1.675 \pm 0.191$ \\
144334 & $3.10 \pm 0.10$ & $1.361 \pm 0.321$ & $0.938 \pm 0.254$ & $0.701 \pm 0.191$ \\
142301 & $1.80 \pm 0.31$ & $1.417 \pm 0.409$ & $0.968 \pm 0.209$ & $0.728 \pm 0.151$ \\
142250 & $3.10 \pm 0.10$ & $0.896 \pm 0.328$ & $0.654 \pm 0.287$ & $0.433 \pm 0.190$ \\
146001 & $4.04 \pm 0.38$ & $1.804 \pm 0.362$ & $1.467 \pm 0.226$ & $1.216 \pm 0.184$ \\
145792 & $4.87 \pm 0.39$ & $2.944 \pm 0.417$ & $2.221 \pm 0.302$ & $1.569 \pm 0.214$ \\
144661 & $3.10 \pm 0.10$ & $1.612 \pm 0.321$ & $1.177 \pm 0.267$ & $0.840 \pm 0.191$ \\
145483 & $3.10 \pm 0.10$ & $1.054 \pm 0.332$ & $0.655 \pm 0.244$ & $0.504 \pm 0.188$ \\
144844 & $3.31 \pm 0.43$ & $1.911 \pm 0.324$ & $1.353 \pm 0.260$ & $1.024 \pm 0.200$ \\
142884 & $2.11 \pm 0.24$ & $2.121 \pm 0.374$ & $1.476 \pm 0.212$ & $1.173 \pm 0.167$ \\
146416 & $3.10 \pm 0.10$ & $1.309 \pm 0.331$ & $0.831 \pm 0.244$ & $0.642 \pm 0.188$ \\
142315 & $4.79 \pm 0.57$ & $1.522 \pm 0.392$ & $1.108 \pm 0.229$ & $0.911 \pm 0.185$ \\
145102 & $4.29 \pm 0.42$ & $1.964 \pm 0.416$ & $1.406 \pm 0.230$ & $1.183 \pm 0.191$ \\
\hline & & & & \\
\hline
\end{tabular}


TABLE 6

DeREDdened Luminosities

\begin{tabular}{cccc}
\hline \hline HD & $\begin{array}{c}L_{0}[973 \AA] \\
\left(\text { counts s }^{-1} \text { bandpass }^{-1}\right)\end{array}$ & $\begin{array}{c}L_{0}[1106 \AA] \\
\left(\text { counts s}^{-1} \text { bandpass }^{-1}\right)\end{array}$ & $\begin{array}{c}L_{0}[1261 \AA] \\
\left(\text { counts s }^{-1} \text { bandpass }^{-1}\right)\end{array}$ \\
\hline 149438 & $10245.66 \pm 3441.07$ & $10425.00 \pm 2562.57$ & $4868.16 \pm 888.83$ \\
143275 & $25820.66 \pm 7523.29$ & $28877.76 \pm 6605.25$ & $8562.69 \pm 1553.04$ \\
144217 & $22075.63 \pm 5718.65$ & $24581.37 \pm 4746.53$ & $6592.99 \pm 987.86$ \\
143018 & $12639.08 \pm 4245.47$ & $15448.11 \pm 3798.14$ & $3865.27 \pm 706.00$ \\
147165 & $24788.61 \pm 5397.26$ & $34514.40 \pm 6397.50$ & $11254.84 \pm 1837.60$ \\
142669 & $1154.06 \pm 387.59$ & $1636.54 \pm 402.26$ & $812.09 \pm 148.26$ \\
144470 & $6821.63 \pm 1717.75$ & $9307.61 \pm 1946.01$ & $3750.34 \pm 667.08$ \\
145502 & $9356.78 \pm 2939.32$ & $9679.58 \pm 2302.78$ & $2161.15 \pm 408.95$ \\
145482 & $925.42 \pm 310.82$ & $1591.84 \pm 391.31$ & $552.94 \pm 100.97$ \\
148605 & $1020.50 \pm 342.79$ & $1591.08 \pm 391.21$ & $732.11 \pm 133.73$ \\
141637 & $1369.92 \pm 455.73$ & $2025.88 \pm 388.45$ & $496.32 \pm 72.53$ \\
142114 & $2621.35 \pm 1118.08$ & $3696.17 \pm 803.85$ & $1380.95 \pm 214.42$ \\
147933 & $43881.32 \pm 10957.16$ & $24816.03 \pm 5148.69$ & $12942.74 \pm 2326.49$ \\
148479 & $1613.21 \pm 738.08$ & $2050.59 \pm 516.89$ & $794.95 \pm 134.24$ \\
\hline 148184 & $10002.99 \pm 3585.40$ & $14499.43 \pm 3999.70$ & $3300.90 \pm 873.62$ \\
142096 & $959.72 \pm 337.73$ & $1608.49 \pm 429.98$ & $522.12 \pm 145.48$ \\
142184 & $632.95 \pm 224.99$ & $1060.83 \pm 288.53$ & $208.64 \pm 59.45$ \\
142990 & $98.07 \pm 33.11$ & $259.12 \pm 67.95$ & $110.27 \pm 30.11$ \\
142165 & $65.45 \pm 22.60$ & $208.89 \pm 57.02$ & $72.51 \pm 19.49$ \\
142378 & $42.96 \pm 14.80$ & $137.09 \pm 37.27$ & $73.45 \pm 18.26$ \\
142883 & $331.62 \pm 115.84$ & $606.69 \pm 162.06$ & $128.96 \pm 35.15$ \\
144334 & $0.24 \pm 0.08$ & $6.23 \pm 1.63$ & $43.57 \pm 11.90$ \\
142301 & $0.24 \pm 0.08$ & $6.21 \pm 1.61$ & $43.35 \pm 10.71$ \\
142250 & $11.22 \pm 3.78$ & $46.41 \pm 12.17$ & $26.00 \pm 7.10$ \\
146001 & $6.33 \pm 2.20$ & $38.75 \pm 10.56$ & $43.65 \pm 11.75$ \\
145792 & $35.59 \pm 12.48$ & $113.58 \pm 31.76$ & $61.12 \pm 17.70$ \\
144661 & $1.75 \pm 0.60$ & $14.43 \pm 3.78$ & $29.31 \pm 8.00$ \\
145483 & $0.01 \pm 0.00$ & $1.22 \pm 0.31$ & $13.26 \pm 3.62$ \\
144844 & $0.28 \pm 0.10$ & $7.30 \pm 1.95$ & $29.85 \pm 8.35$ \\
142884 & $0.02 \pm 0.01$ & $1.39 \pm 0.36$ & $6.10 \pm 3.89$ \\
146416 & $0.00 \pm 0.00$ & $0.54 \pm 0.14$ & $8.58 \pm 2.31$ \\
142315 & $0.13 \pm 0.05$ & $3.47 \pm 0.97$ & $7.83 \pm 2.14$ \\
145102 & $0.01 \pm 0.00$ & $0.78 \pm 0.21$ & \\
\hline
\end{tabular}




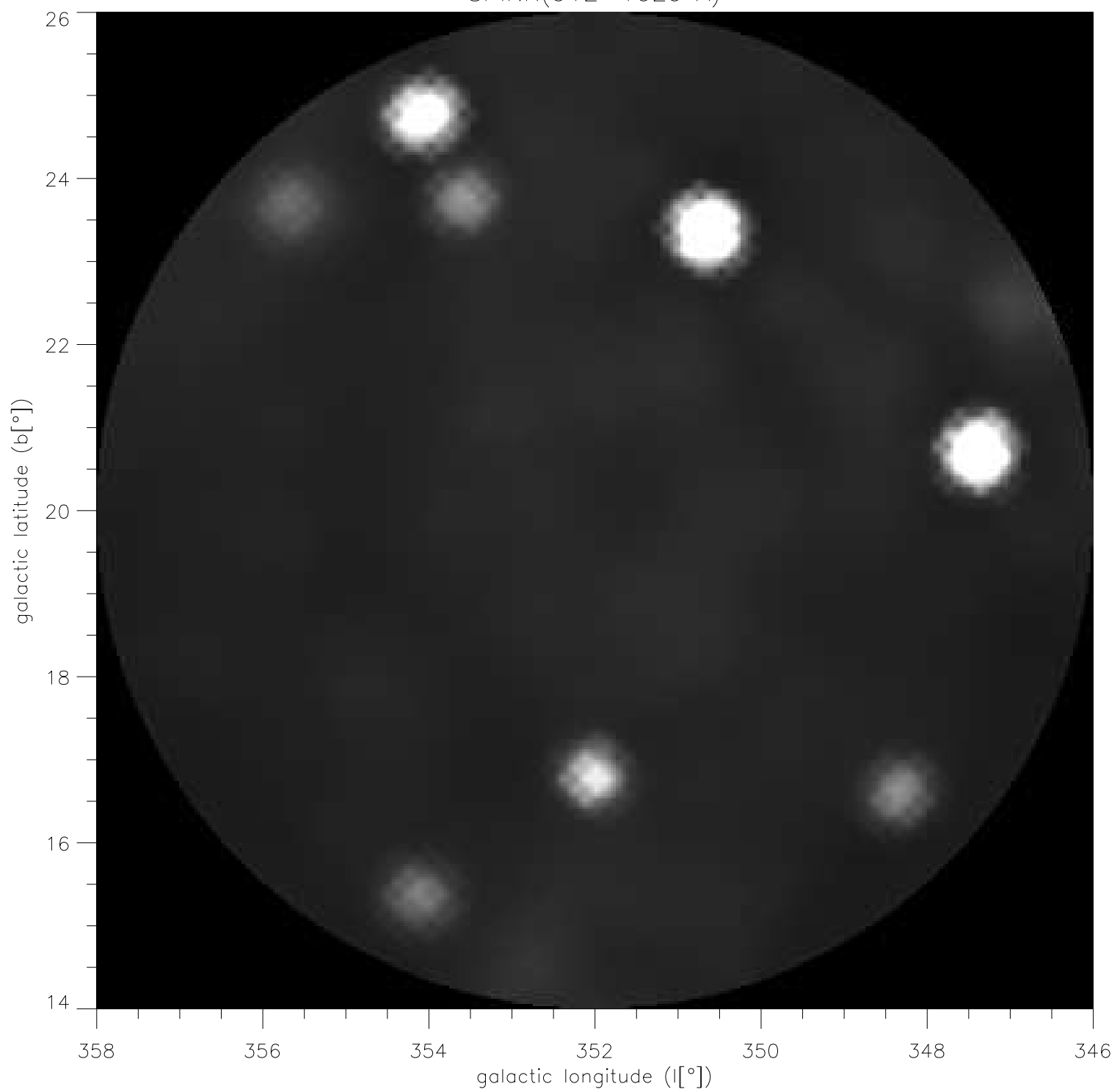




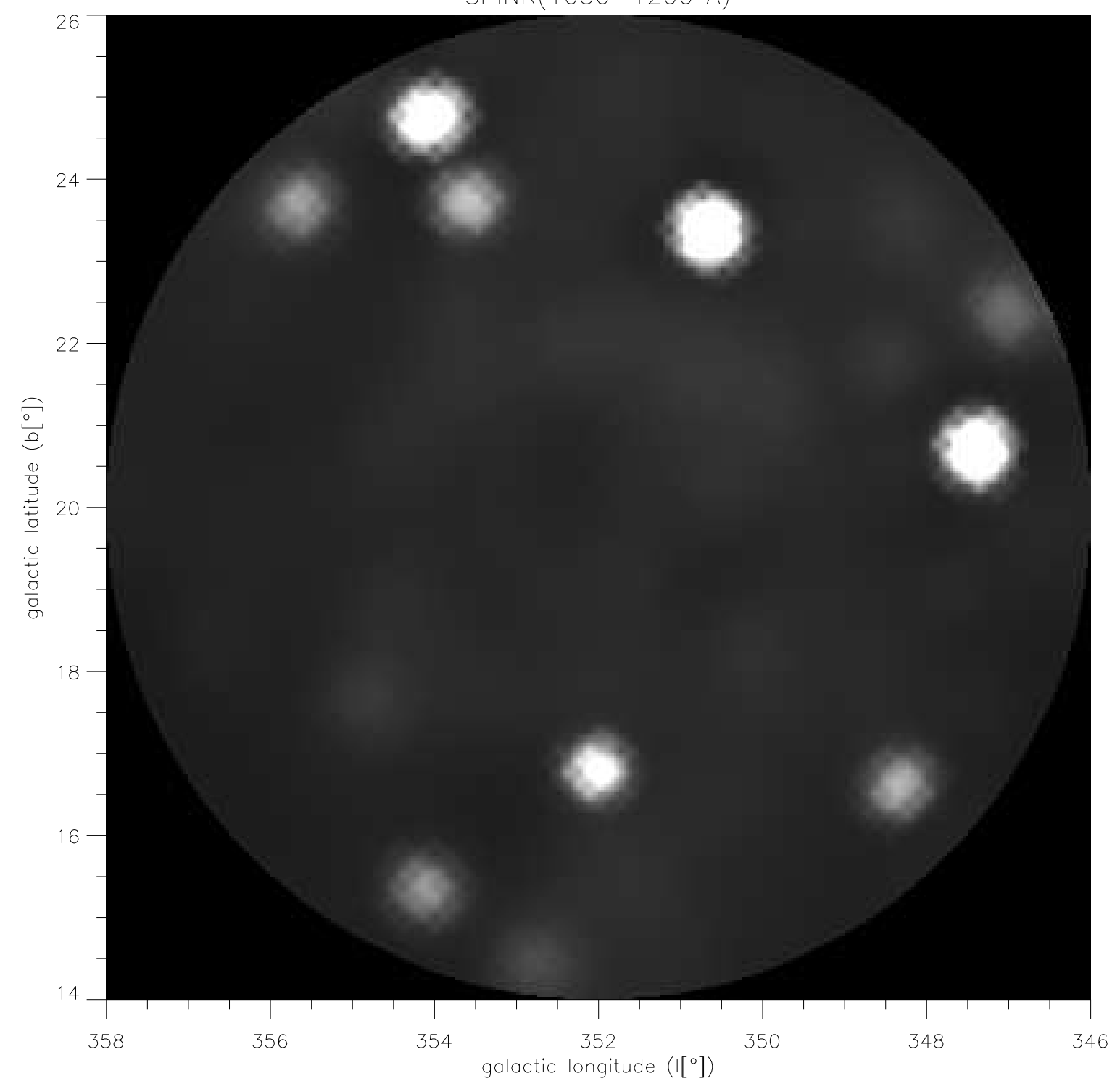




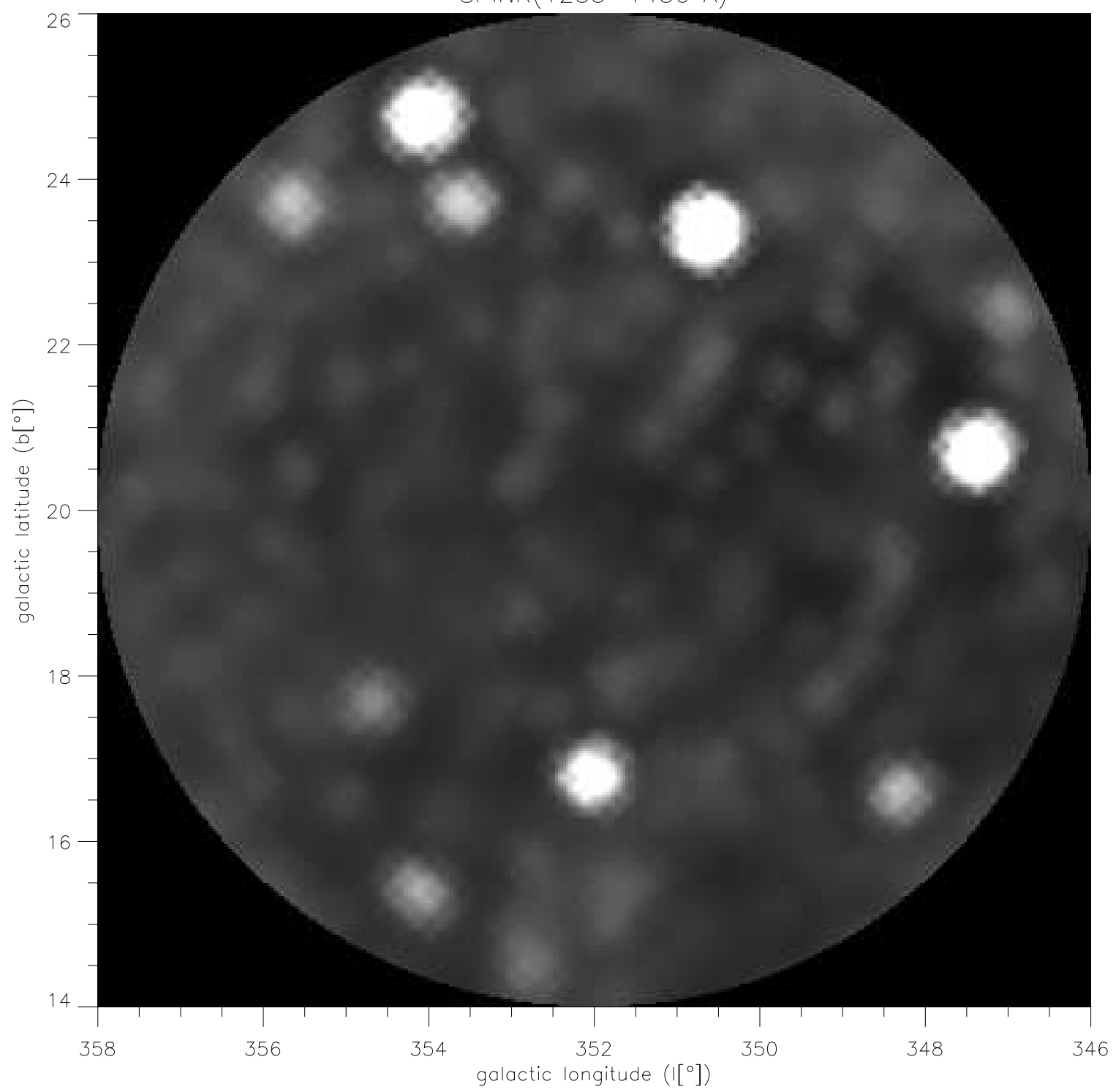




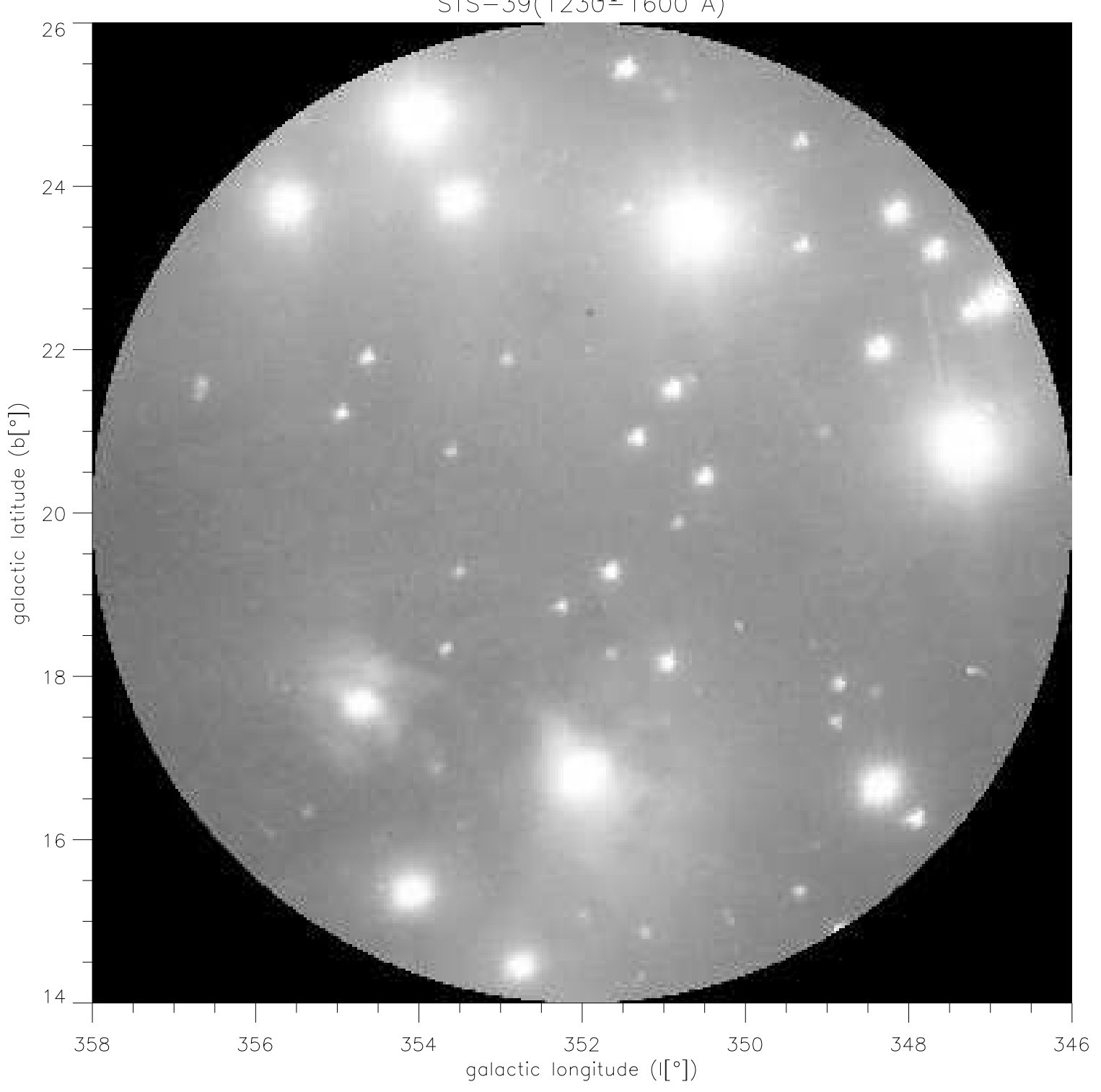

FIG. 1. - Comparison of broadband (1230-1600 ̊) image taken with the STS-39 FUVCam (100s exposure) and the reconstructed images in each of the SPINR wavelength bands. Although the spatial resolution is lower in the SPINR images than the STS-39 FUVCam image, the basic structures of region are clearly visible in the 1235-1450 A bandpass image and in close correspondence with the STS-39 FUVCam image for 1230-1600 $\AA$. The reconstructed SPINR images are included for visualization purposes only. All quantitative analysis is done using the raw SPINR sinogram data. 


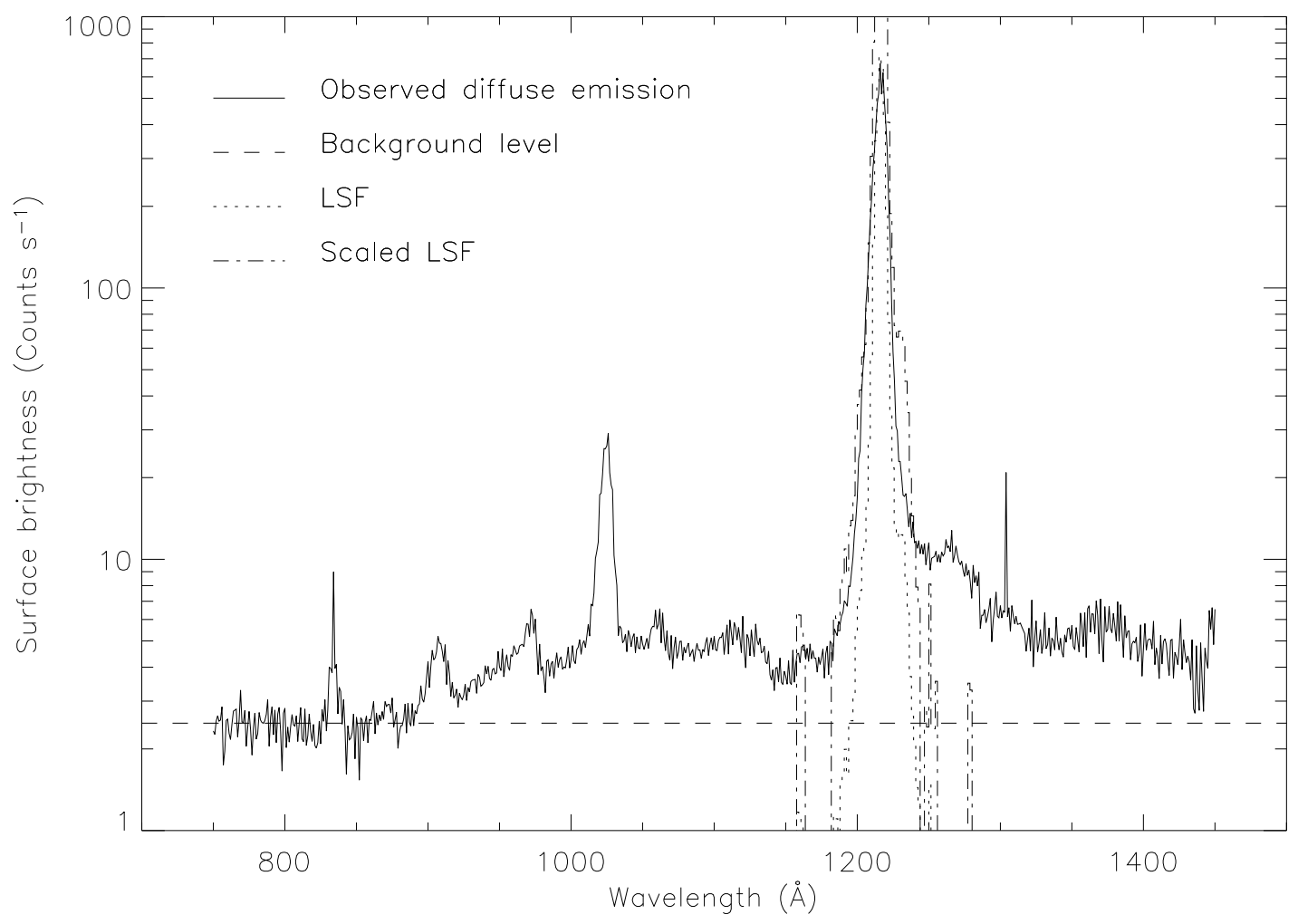

FIG. 2.- The raw observed diffuse brightness and the derived background level. In addition to the background level (which is a combination of the detector dark rate and scattered Ly $\alpha$ ) this figure shows the approximate Ly $\alpha$ LSF. The LSF is from calibration data shifted in wavelength and scaled to match the observed emission line strength. A second LSF is shown scaled to the strength appropriate for the wings of Ly $\alpha$ as they impinge on the high sensitivity areas of the detector (away from the core of Ly $\alpha$ ). For further details of the detector sensitivity see Paper I. 


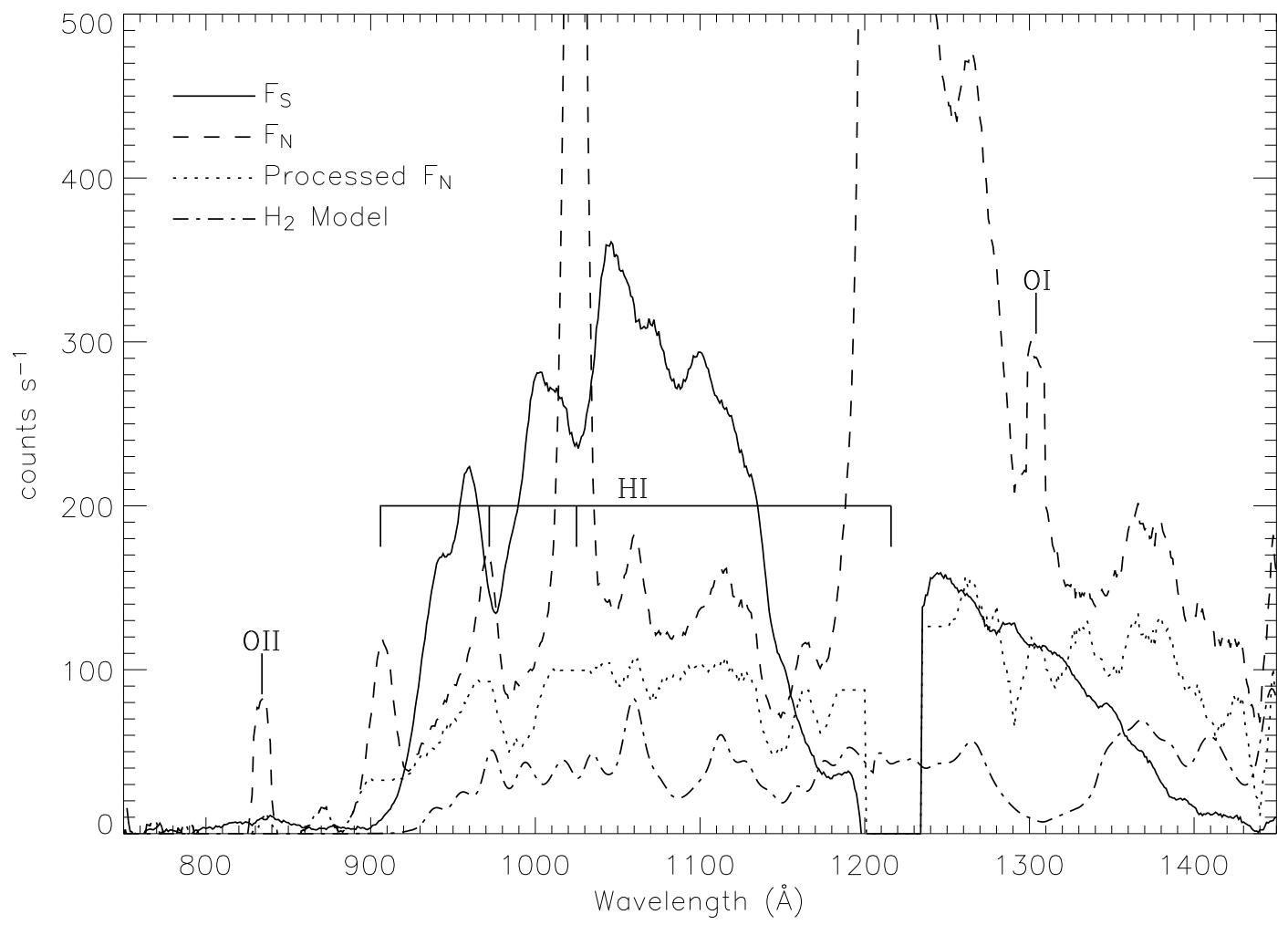

FIG. 3.- Comparison of the nebular (dotted line) and stellar (solid line) spectra derived from SPINR data. Telluric lines that were removed from the unprocessed nebular (dashed line) spectrum are noted. Also, the modeled $\mathrm{H}_{2}$ emission removed from nebular spectrum is presented (dash-dot line). The nebular spectrum remains relatively constant across all bandpasses, while the stellar spectrum peaks in the 1030-1200 ^ bandpass. This directly corresponds to derived nebular to stellar flux ratios (Table 2), which shows a steady increase in

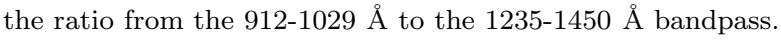




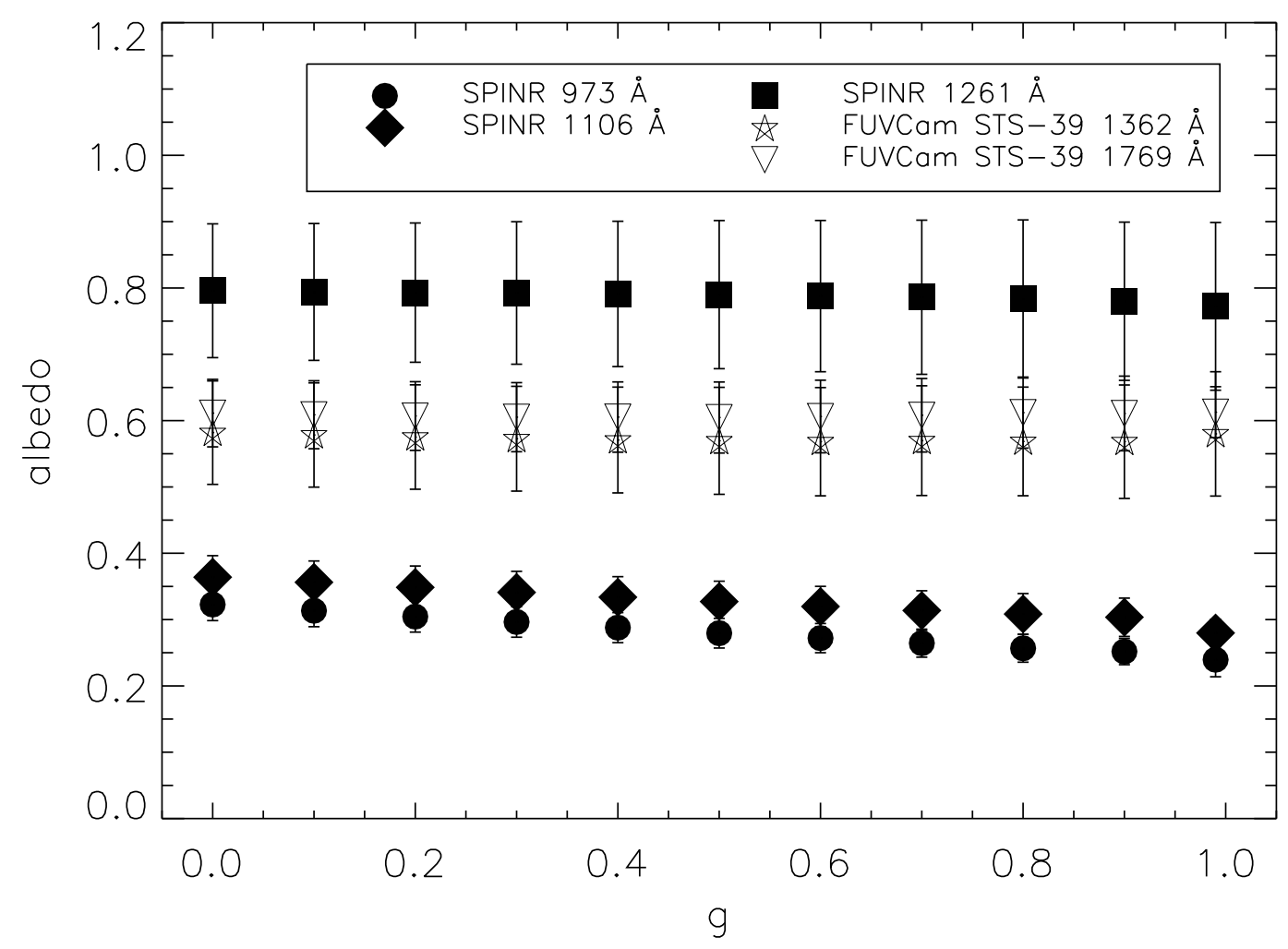

FIG. 4.- Optimal dust albedo values as a function of $g$ for the SPINR and STS-39 FUVCam data. As can be seen there is very little variation in the derived values of $a$ across all values of $g$. This is a direct result of the spherical cloud with embedded stellar sources geometry of the Upper Scorpius region. 


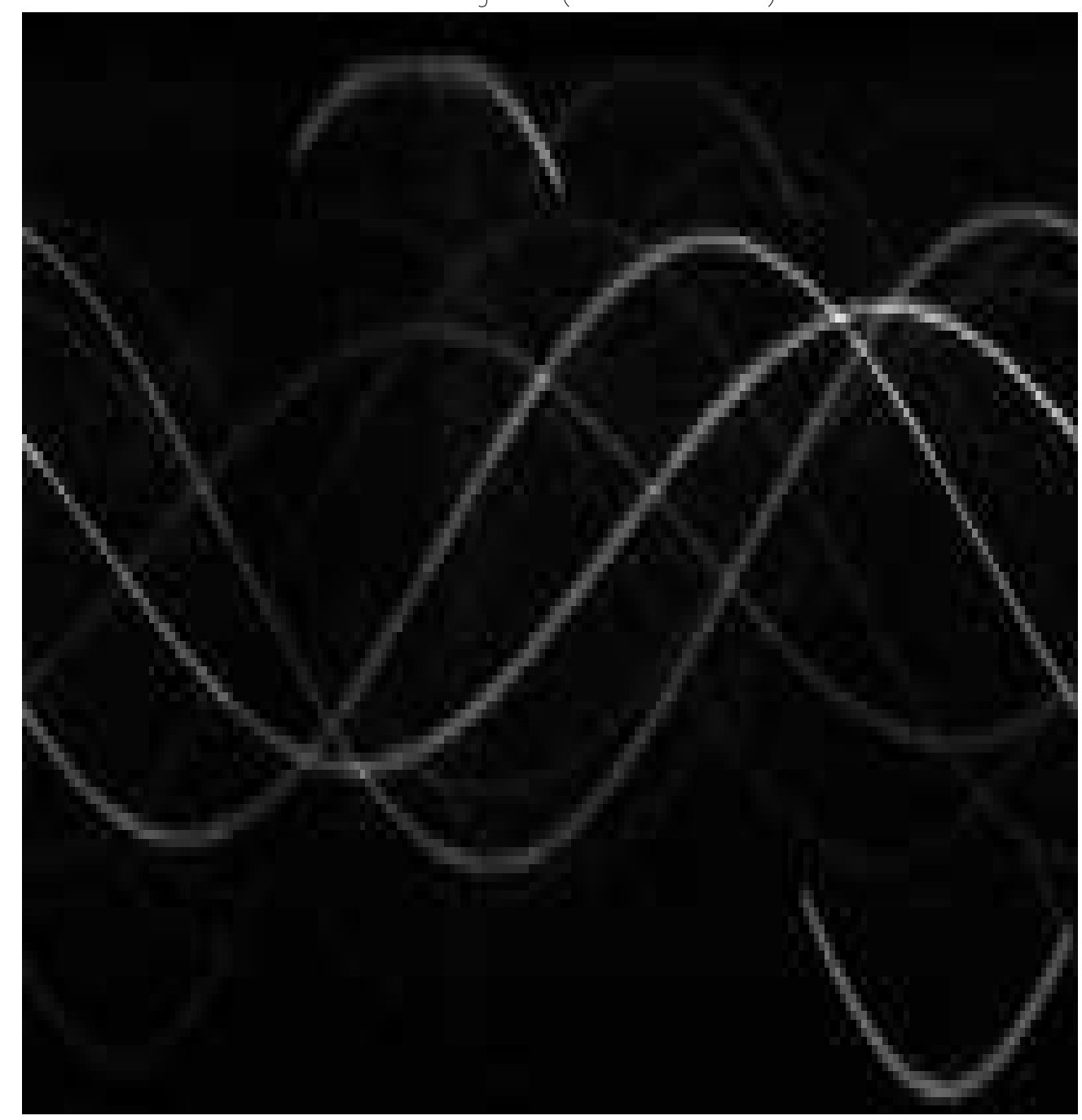


SPINR Image $(1030-1200 \AA)$

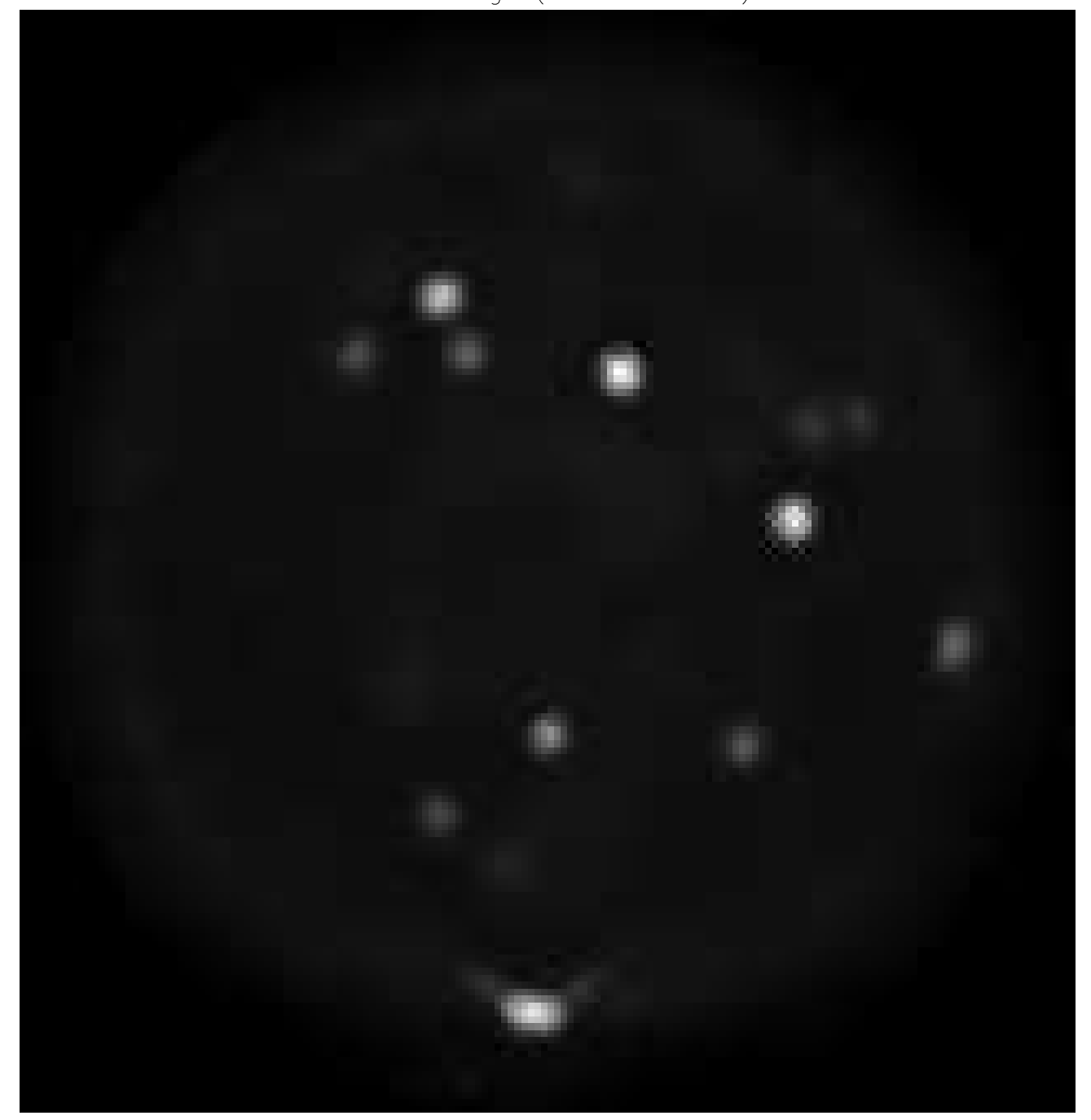


Model Sinogram $\left(a=0.30, g=0.96, \lambda_{\text {eff }}=1106 \AA\right)$

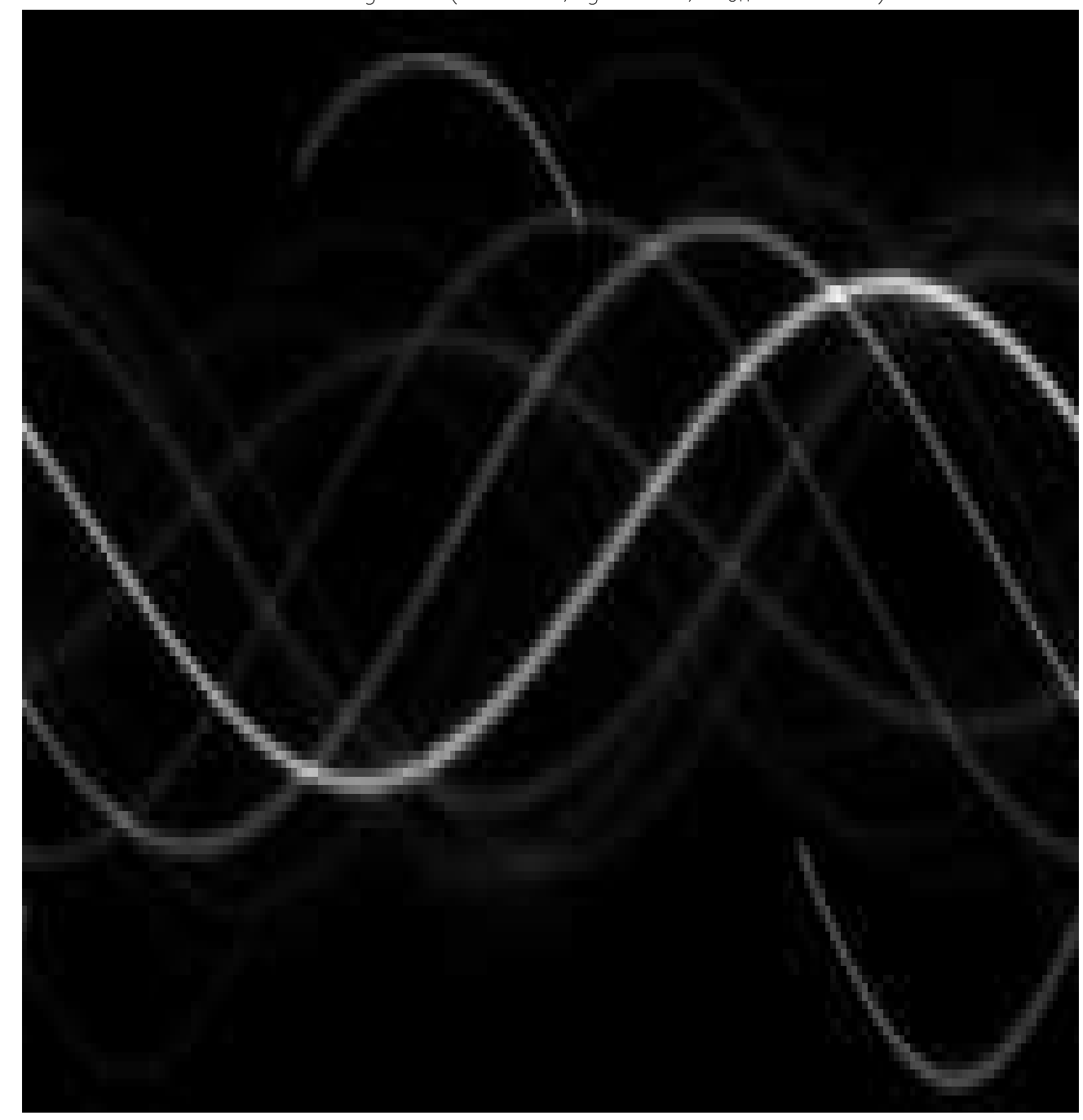


Model Image $\left(a=0.30, g=0.96, \lambda_{\text {eff }}=1106 \AA\right)$

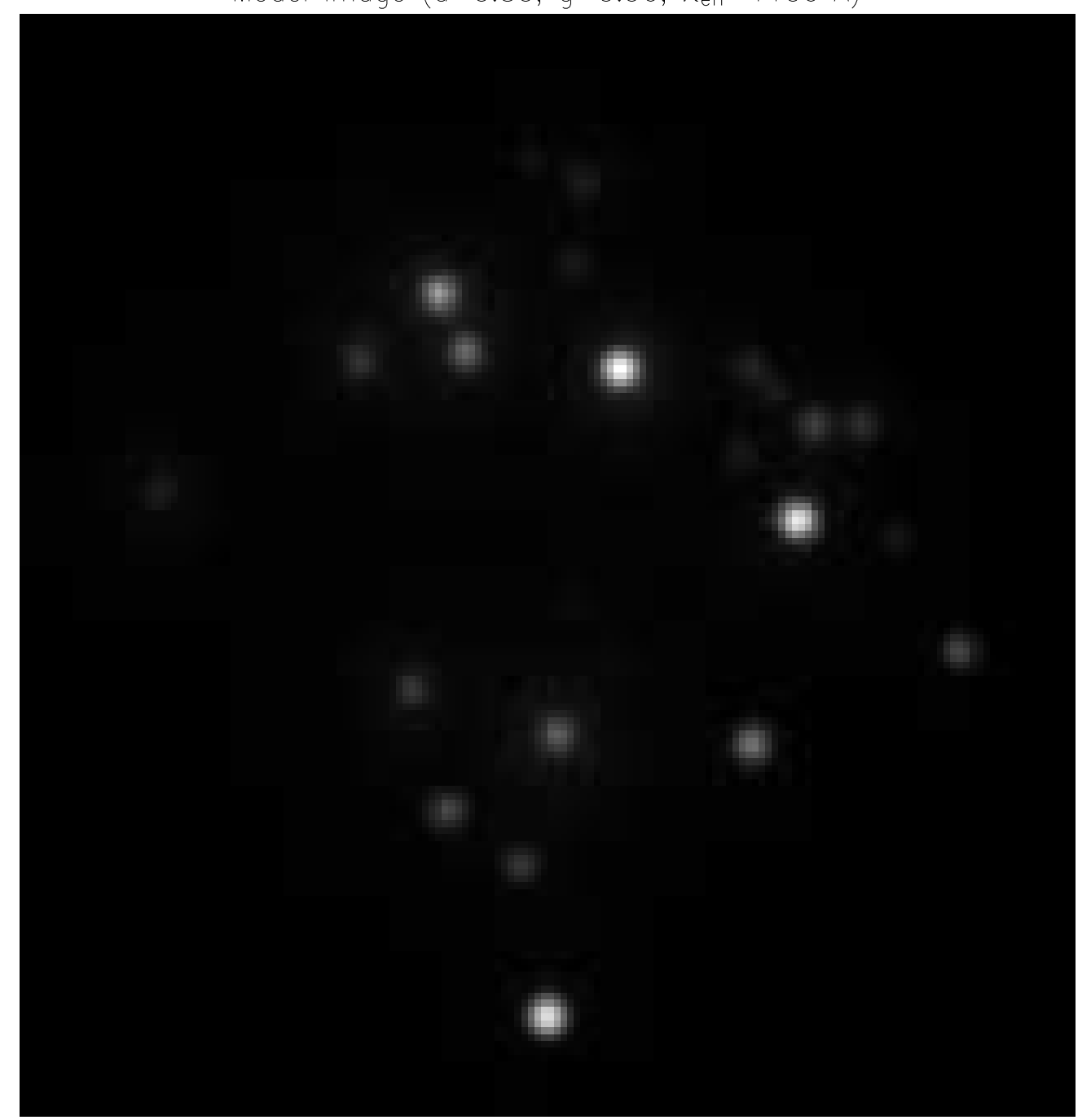


Model Sinogram $\left(a=0.75, g=0.0, \lambda_{\text {eff }}=1106 \AA\right)$

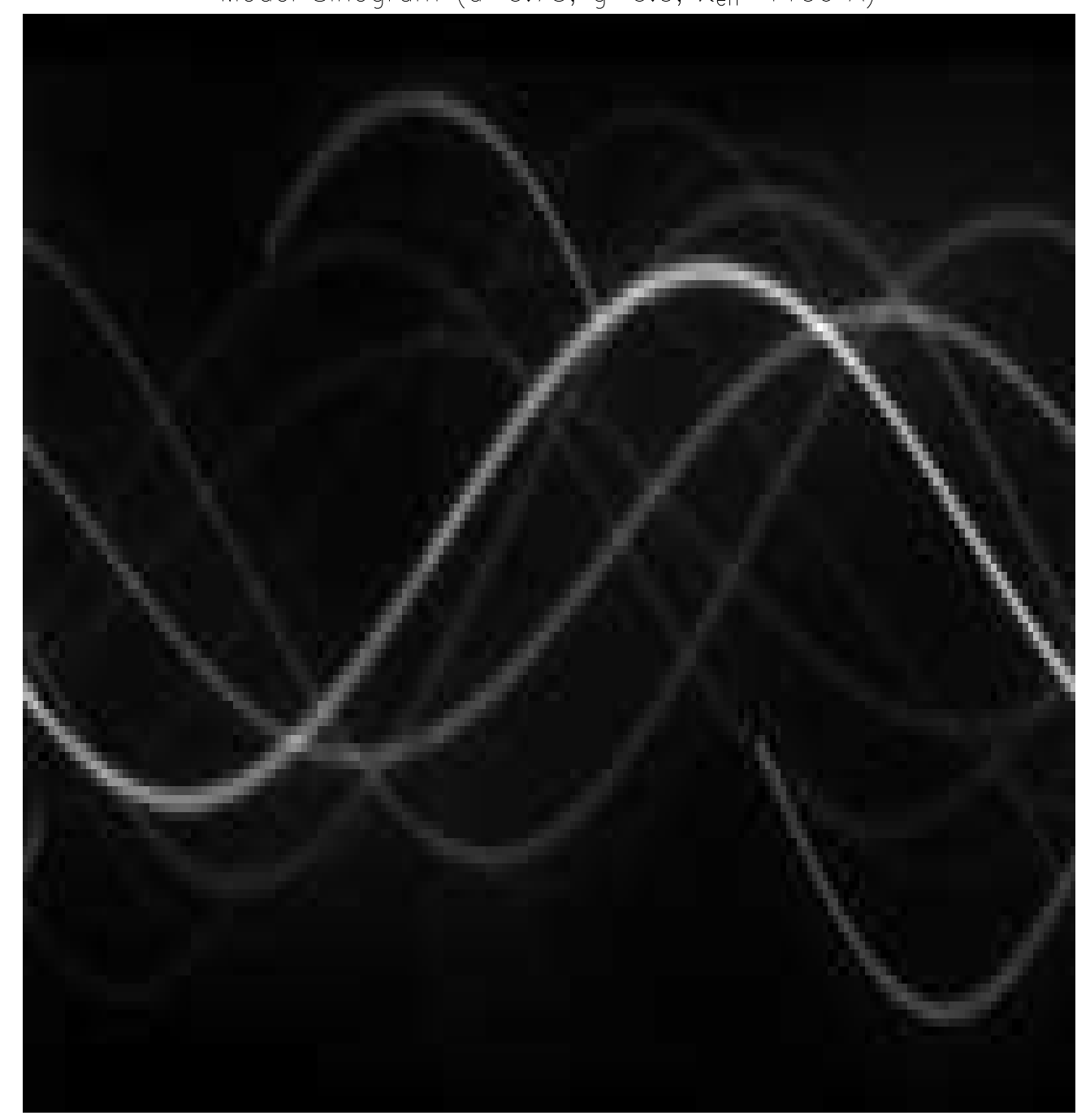




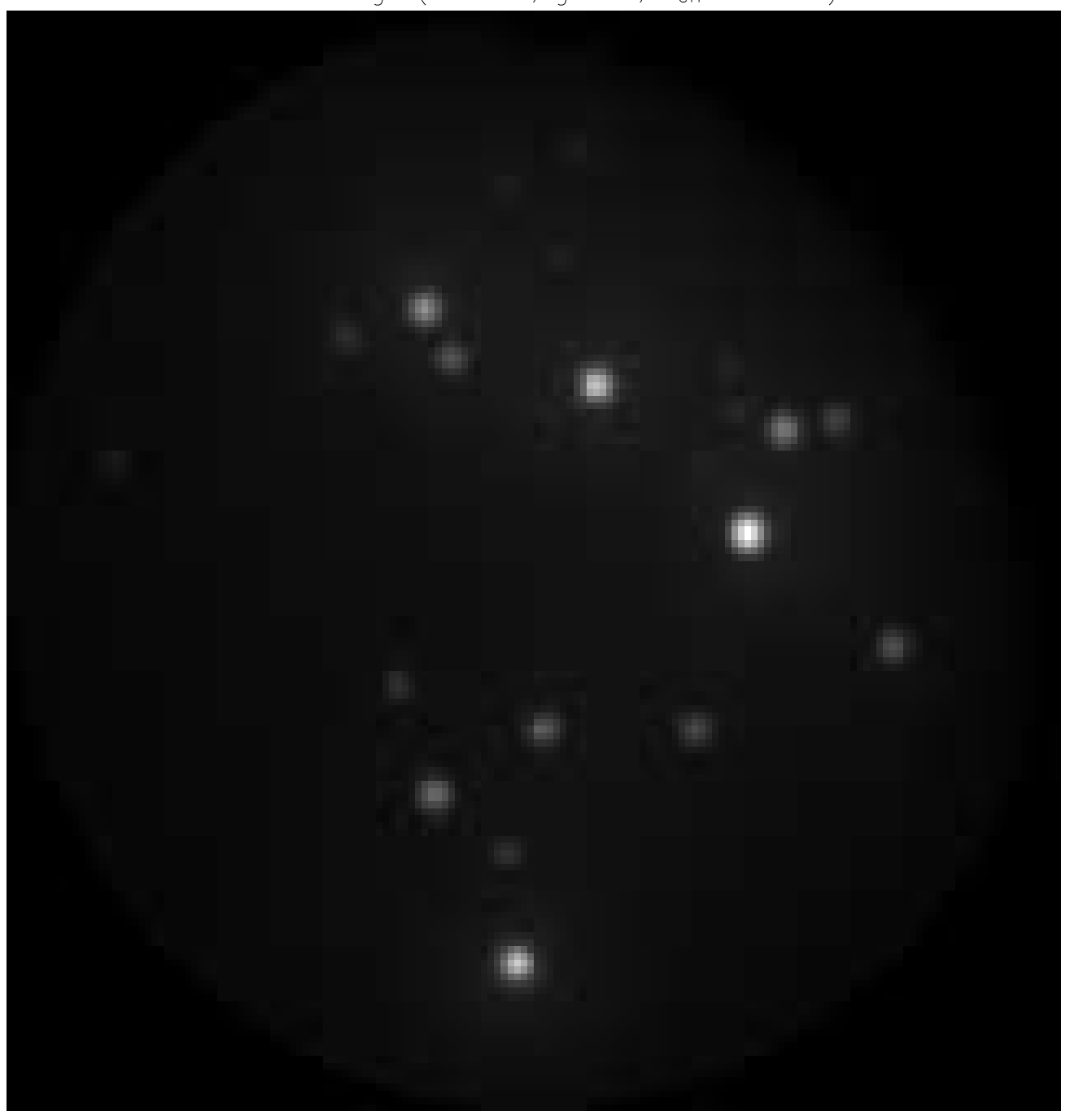

FIG. 5. - Comparison of the SPINR data, best fit dust model, and a model outside the acceptable ranges for $a$ and $g$ for the 1030-1200 $\AA$ bandpass. The cross-correlation metric between the sinograms was used in determining the optimal $g$ value and the uncertainty in this measurement for the 1030-1200 $\AA$ bandpass. Model/observation comparisons were made in sinogram space because the transformation from image to sinogram space is more precisely known than the transformation from sinogram to image space. The signal-to-noise ratio is higher for the model, which accounts for the small differences seen between the images. The $a=0.75$ and $g=0.0$ model is brighter in the regions between the stellar sources, which is not generally seen in the SPINR observations. 


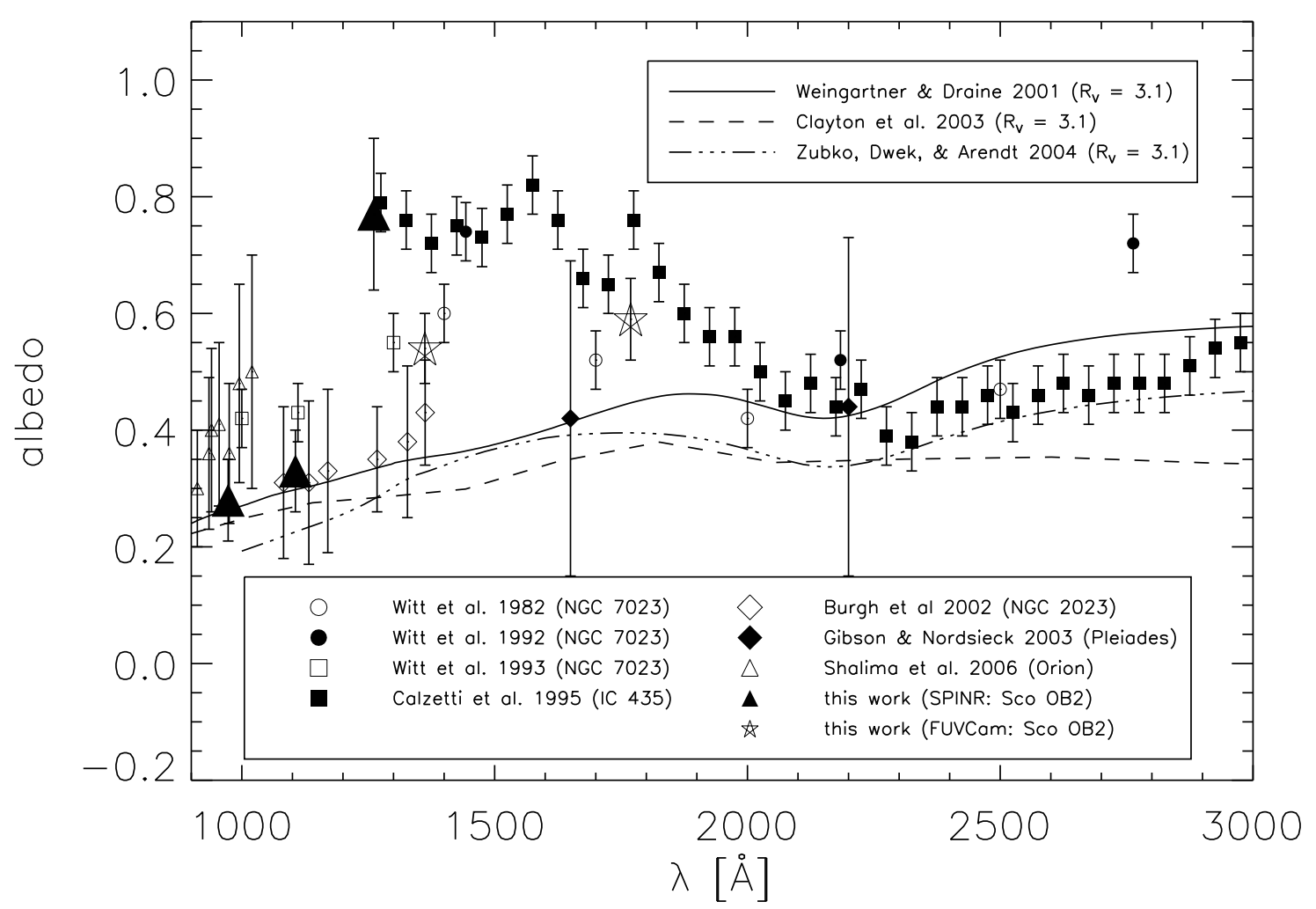

FIG. 6.- A plot of albedo (a) vs. wavelength for the values derived in this study along with other values from literature for reflection nebulae. The values for the albedo derived in this study show strong correspondence with albedo values derived from other observational studies. The derived albedos for $\lambda_{e f f}=973 \AA$ and $1106 \AA$ are well correlated with the results from Calzetti et al. (1995) and the dust model of Weingartner \& Draine (2001). The derived albedo for $\lambda_{e f f}=1261 \AA$ is higher than would nominally be expected from the STS-39 FUVCam observations, but still within $1 \sigma$ of other albedo measurements in this spectral region, which all show significant deviation from model predictions. 